\title{
Gradhiva
}

\section{Anthropologie d'un tube des Années folles}

De jazz en littérature

Anthropology of a hit from the Roaring Twenties, from jazz to litterature

Jean Jamin et Yannick Séité

\section{CpenEdition}

Journals

Édition électronique

URL : http://journals.openedition.org/gradhiva/566

DOI : $10.4000 /$ gradhiva.566

ISSN : 1760-849X

Éditeur

Musée du quai Branly Jacques Chirac

Édition imprimée

Date de publication : 1 novembre 2006

Pagination : 5-33

ISBN : 2-915133-44-1

ISSN : 0764-8928

Référence électronique

Jean Jamin et Yannick Séité, "Anthropologie d'un tube des Années folles », Gradhiva [En ligne], 4 |

2006, mis en ligne le 16 décembre 2009, consulté le 10 décembre 2020. URL : http://

journals.openedition.org/gradhiva/566 ; DOI : https://doi.org/10.4000/gradhiva.566

Ce document a été généré automatiquement le 10 décembre 2020.

(c) musée du quai Branly 


\title{
Anthropologie d'un tube des Années folles
}

\author{
De jazz en littérature* \\ Anthropology of a hit from the Roaring Twenties, from jazz to litterature
}

\author{
Jean Jamin et Yannick Séité
}

1 Objet musical parmi les plus répandus au monde, la chanson relève d'un genre d'expression artistique singulier dont la définition et, par conséquent, les contours posent de sérieux problèmes aux musicologues (Fabri 2003 : 675), sans parler de la distance, voire du détachement que manifestent, quant à eux, les anthropologues et ethnomusicologues face à son étude, comme si la familiarité, la brièveté, la structure métrique et le schéma circulaire (couplet/refrain) de cet objet dispensaient de s'interroger méthodiquement sur la place et la fonction qu'il occupe dans toute société humaine, tant du point de vue de la mémoire que de la sensibilité collectives. Ce qui va de soi est sans doute ce qui se comprend le moins. Car, si l'on s'accorde sur ce qu'est une chanson, sur le fait de reconnaître que ce qu'on écoute ou chantonne hic et nunc relève bien du genre " chanson ", il est par contre extrêmement difficile de dire de quoi elle est faite et à quoi elle renvoie très précisément, en somme ce qu'elle traduit ou signifie.

2 Que «tout finisse par des chansons", suivant le refrain bien connu du vaudeville $d u$ Mariage de Figaro, suffirait-il à reléguer celles-ci dans la sphère de la futilité ou de la frivolité, si ce n'est de la trivialité, et, ce faisant, à les refouler au bas des hiérarchies culturelles et artistiques, les privant ainsi de toute légitimité épistémologique? En quelque sorte art mineur plutôt qu'art moyen. Mais en quoi, par exemple, seraient-elles différentes à ce point de ces compositions pour voix et instrument que sont ce que, depuis Hector Berlioz, il est convenu d'appeler dans la tradition musicale classique les mélodies? Serait-ce que les chansons proprement dites fussent sinon dépourvues de mélodies du moins vouées à n'en proposer que des formes simplifiées, frustes ou même dégradées ? De sorte que, peut-être bonnes à entendre ou à fredonner, les chansons ne seraient guère bonnes à penser. Elles le seraient d'autant moins que leurs création, diffusion et circulation semblent dépendre, notamment dans notre culture, de la pression des modes 
et de l'intervention des médias tout autant que des stratégies mercantiles et, partant, versatiles du show business (Bonnieux éd. 2004 : 12). Condition quelque peu paradoxale de leur popularité si l'on entend par là qu'elles ressortissent au genre populaire dont l'opinion courante voudrait qu'il fût fait a contrario d'enracinement, de régularité, de stabilité, de répétition, en un mot de folklore. En fonction du marché des biens culturels, les chansons se trouveraient destinées à passer comme passent les couleurs du temps. Est-ce pour cette raison qu'on les catalogue aujourd'hui sous la rubrique "variétés » ou que, jadis, on les rangeait dans la catégorie «musiques légères»? Le fait est que leur structure fermée et cyclique ne les empêche nullement de devenir des yoyos de la mémoire ou de l'histoire, non plus que ce qu'on nomme depuis le début des années 1960 des tubes, par où justement s'engouffre ou s'échappe de l'air - symbole de l'éphémère.

Signalons un autre écueil dans leur approche : la dimension narrative, topique ou réaliste que l'on prête parfois aux chansons est tout sauf évidente, quand bien même leurs paroles en viendraient-elles à raconter une histoire, évoquer des lieux, des saisons, des instants, effeuiller des vies, détailler des drames, dénoncer des régimes ou des institutions, moquer des classes, des castes et des étiquettes, exprimer des aspirations... ou des regrets (Jamin 2004, 2006 ; Lortat-Jacob 2006). Mais la chanson ne se réduit pas seulement à ses paroles : celles-ci peuvent devenir incompréhensibles ou absurdes, s'oublier, se détourner, s'altérer, se décomposer (le scat singing en est un exemple limite ${ }^{1}$ ), sans que disparaisse le pouvoir d'enchantement de leur modulation, s'efface le souvenir du timbre de voix de leur interprète, s'estompe leur aspect lancinant, s'amenuisent le désir et le plaisir de fredonner leur air. Les "îlots référentiels » qui surgissent çà et là dans des chansons semblent n'avoir d'autre alternative que de se nier en tant que tels. On ne peut parler à leur propos, et s'agissant de leur contenu, qu'il soit musical ou narratif, d'« effets de réel ». Tout au plus peuvent-elles être parfois utilisées comme référents, autrement dit comme rouages d'une technique romanesque (nous le verrons dans La Nausée de JeanPaul Sartre) venant renforcer une couleur locale, une atmosphère, ou la véracité de telle ou telle scène racontée (Sabatier 2004:65 et suiv.). Aussi serions-nous tentés de les considérer comme des sortes de sémaphores acoustiques qui contribuent à baliser, orienter et ordonner le champ des émotions collectives, si ce n'est même celui de l'inconscient collectif. Alain (1963: 135) remarquait qu'un simple titre de chanson pouvait prendre "puissance de poème ", mais un " poème en action » au sens où la mélodie qu'il évoque ramène le mouvement des passions qui l'a fait naître "au mouvement même qui les guérit " en l'écoutant (ibid.: 135). On serait tenté d'ajouter, suivant en cela Daniel Percheron (1994: 205), que rien qu'un titre de chanson a aussi "pouvoir de résurrection ». Loin donc d'être pure nostalgie ou écho ductile d'un passé révolu, la chanson a quelque chose d'inchoatif qui la soustrait au temps qui passe, peut-être parce qu'elle devient elle-même l'image sonore d'un autre temps qui n'est ni celui de la mémoire ni celui de la chronologie, et que l'on désirerait voir et sentir se boucler pour le vivre de nouveau, comme le temps des cerises. La chanson rappelle le temps qu'elle chante; son air chante (enchante) le lieu où elle chante et se chante (Hollier $1982: 207$ ) - un temps qui oscille constamment, ne serait-ce que par le rythme, entre la linéarité (mélodie) et la verticalité (harmonie). Chacun de nous en a certainement fait l'expérience : des airs nous trottent par la tête, se murmurent sur nos lèvres, mais sans qu'on sache dire comment et pourquoi ils nous hantent et continuent de nous hanter, non plus qu'expliquer leur soudaine et déconcertante apparition, venus tantôt du fond de l'enfance ou des âges, tantôt des membranes d'un haut-parleur, à présent des vibrations d'un casque de baladeur. Pour reprendre la question qui avait présidé à la journée d'étude dont cet 
article est issu, il convient de s'interroger justement sur ce qu'il y a dans un air. Est-ce, comme on le dit communément, et pas si naïvement, l'«air du temps»? Mais de quel temps cet air est-il fait? Ne serait-il, comme le suggère le titre du livre de Bernard Pouchèle et Bernard Lachat (2006), qu'un «bruit de fond de l'histoire»? Ou bien ne traduirait-il que les turbulences de la société ou, plus intimement, ne brasserait-il que l'écume de la mémoire et de la sensibilité?

On fera l'hypothèse que la manière dont une société chante et se chante le monde permet d'accéder à la formation, la canalisation et la reproduction des émotions, et constitue de ce fait un opérateur anthropologique puissant, quoique oblique, qui ouvre des fenêtres sur les représentations au sens strict de l'identité, de l'altérité, de l'intimité et de la sensibilité. En effet, il se joue là, dans et par la chanson, qu'elle soit berceuse ou romance, ritournelle ou ballade, pastourelle ou « tragédie instantanée » (Jean-Paul Sartre), tout un mode d'articulation entre le social et l'intime, l'individuel et le collectif, le public et le privé, la mémoire et l'émoi. Égrener les notes d'une chanson, suggère Michel Leiris (1988: 105), est une façon de revenir à soi en empruntant le plus court chemin, mais où, quels qu'en soient les méandres, on finit toujours par croiser celui tout aussi court qu'empruntent les autres.

5 Cet article se propose donc, à partir de la musique de jazz et de l'un de ses songs - lesquels constituent une partie non négligeable du répertoire des jazzmen (Baudoin 1994a; Williams 2006) -, d'explorer cette sorte d'« épopée de la chose » que sont, selon la belle formule d'Alain (1963: 136), musique et chant, et où ce qui s'exprime dans leur apparente contingence est non seulement une modulation mais un modelage du rapport de soi à soi, et de soi à l'autre. Sujet bien évidemment anthropologique que nous aborderons à travers l'étude de trois œuvres de la littérature contemporaine où le « chant du jazz », que toutes trois placent au premier plan, traduit bien cette dialectique de l'identité et de l'altérité en jouant sur des associations et des contrastes de couleurs tout autant musicales que charnelles, à l'image de la célèbre suite Black, Brown and Beige que Duke Ellington créa le 23 janvier 1943 au Carnegie Hall de New York ${ }^{2}$. Vingt ans plus tôt, presque jour pour jour, c'est un autre musicien - celui-ci européen et de formation classique - qui, au terme d'un court séjour dans cette même ville, fit part de sa découverte d'un alliage inouï de sons et de couleurs, de peaux et de voix, d'un corps-à-corps inédit de la mesure et de la syncope. À bord du paquebot Rochambeau le ramenant en France, Darius Milhaud s'enthousiasmait encore, dans une lettre adressée à son ami Henri Hoppenot et datée du 20 février 1923 (Milhaud et Hoppenot 2005 : 68), d'avoir entendu là, à Harlem, dans de simples estaminets remplis de Noirs, des « jazz populaires magnifiques », des " danses et chants de Négresses aux voix cuivrées ", des combinaisons inusitées de rythmes, de timbres et d'accords qui allaient le hanter pendant une bonne partie de l'année et même au-delà... Dans une boutique de Harlem, il s'était procuré toute une collection de disques du label Black Swan (première compagnie d'édition phonographique noire-américaine, dont le pianiste et chef d'orchestre Fletcher Henderson était depuis 1921 le directeur musical). Ils devaient l'inspirer pour la composition et l'instrumentation (cuivres, anches, percussions) de la musique de La Création du monde que lui avait commandée Rolf de Maré, le fondateur et directeur de la troupe des Ballets suédois. Dans ce "Ballet nègre", dont l'argument s'inspirant d'une légende africaine fut rédigé par Blaise Cendrars (2005: 463-468) et la chorégraphie réglée par le danseur étoile de la troupe Jean Börlin, Darius Milhaud s'était attaché à combiner accents jazzy (growl, glissando), inflexions bluesy (tierce et septième mineures), rythmes afterbeat et traditions musicales européennes savantes - un 
«intermédiaire", écrivait-il à Hoppenot le 4 août 1923 (ibid.: 73), "entre les phonographes de Broadway et La Passion selon saint Matthieu »-, le tout joué et dansé dans un décor monumental conçu par Fernand Léger, aux longs aplats de couleur noirs, blancs et ocre, où se fondaient les danseurs tels des minstrels ${ }^{3}$. Mais c'est d'un décor en noir, blanc et blond qu'il sera surtout question ici.

\section{Une musique noire jouée par un Blanc}

Dans une nouvelle proche de la parabole, Novecento : pianiste, Alessandro Baricco (1999) place dans la bouche du personnage éponyme cette invective : «Et au cul aussi le jazz ! » Elle est adressée à son partenaire de jeu et rival, le célèbre pianiste créole Jelly Roll Morton au moment où celui-ci, la mine et l'habit chiffonnés, débarque par l'échelle de coupée d'un paquebot transatlantique sur le quai du port de Southampton - personnage qui a bel et bien existét (1885 ou 1890-1941) mais dont, quelques pages plus haut, le portrait brossé par Alessandro Baricco accentue des traits devenus caricaturaux. Coiffé et costumé de blanc immaculé, cravaté, lunetté et chaussé de noir, arborant au doigt « un diamant gros comme ça » (ibid.: 43) et affichant, jusque dans sa mise vernie et sa démarche chaloupée, la suffisance et l'arrogance d'un seigneur des bouges et claques de Storyville d'où il provenait et où il avait joué du piano pendant de longues nuits, Jelly Roll Morton était le seul capable d'envoyer « un blues à faire pleurer un mécano allemand » et de ramasser rien qu'avec des notes effleurées de «ses mains de papillon [...] tout le coton de tous les Nègres du monde» (ibid.: 50). Contre toute attente, Danny Boodmann T.D. Lemon, dit Novecento, le pianiste blanc, si ce n'est blanc-bec par son âge, venait de remporter une superbe victoire sur lui, le pianiste mulâtre quelque peu hâbleur mais dont le toucher était tout de grâce mélodique et de swing, au cours d'une joute musicale endiablée (les jazzmen appellent cela cutting contest) dans les salons du paquebot, devant une foule de rupins en liesse.

7 Tout se passe comme si, par l'invective lancée en guise d'adieu, le jeune Novecento, passager des mers, vainqueur sans élégance ni compassion, avait voulu renvoyer vertement et symboliquement Jelly Roll Morton à ses bas-fonds, à ses années d'apprentissage qui furent surtout de jeux, de combines, de chairs et de stupres. La légende veut en effet que ce soit dans les bordels et bastringues (honky tonks) de La Nouvelle-Orléans qu'il aurait inventé ce qu'on appelle le jazz (Lomax 1950: 41 et suiv.; Armstrong 2006 : 76) - lui-même ayant été plus ou moins (plutôt plus que moins) un vrai flambeur et un souteneur, ce qu'évoque du reste, sans aucune ambiguïté, le surnom « Jelly Roll » (gâteau long et roulé, enrobé de confiture) dont on l'affubla ou qu'il s'attribua.

Dans le film réalisé en 1998 par Giuseppe Tornatore et mis en musique par Ennio Morricone $^{5}$, La Legenda del pianista sull'oceano (La Légende du pianiste sur l'océan) - film naïf au pathos très hollywoodien, mais dont le scénario suit d'assez près la nouvelle d'Alessandro Baricco ${ }^{6}-$, une scène retient l'attention. Dans celle-ci, le narrateur, Tim Tooney (il deviendra le partenaire et l'ami intime du pianiste Novecento) tente de se faire engager comme trompettiste dans l'orchestre de danses, exclusivement formé de musiciens blancs, du paquebot le Virginian qui assure régulièrement la traversée de l'Europe aux Amériques.

9 On est à la fin des années 1920. À Southampton, sur le quai, face à la file des voyageurs et du bureau d'embarquement, Tim Tooney sort son instrument de l'étui, le porte à ses 
lèvres et exécute un ample solo de trompette, coulant, éclatant, éblouissant, à la manière du break de Louis Armstrong dans West End Blues; les badauds, les passagers en partance et le contrôleur de la compagnie de navigation en ont le souffle coupé. Après qu'il a terminé son solo, le contrôleur lui demande : "- C'était quoi ?» Le trompettiste lui répond: «J'en sais rien! - Quand on ne sait pas ce que c'est... alors c'est du jazz!» lui réplique le premier d'un ton graveleux, qui l'embauche sur-le-champ en précisant que ceux d'en haut, c'est-à-dire les premières classes, aimeront ça... Incidemment, la réplique du contrôleur nous plonge au cœur de la question - incidemment, car il ne fait pas de doute que, dans son esprit, cette ignorance, qui n'est cependant pas n'importe laquelle, renvoie autant à la futilité du jazz (musique de divertissement, voire d'ameublement) qu'à son excentricité (musique de la marge) et à son impureté (musique hybride), comme cela avait été souligné par Theodor W. Adorno (2003) dans sa célèbre diatribe contre le jazz en y voyant une "régression de l'écoute musicale» (Béthune 2003; Williams 2005). L'allusion au plaisir que ceux des ponts supérieurs peuvent éprouver laisse penser, par le fait même de l'encanaillement auriculaire auquel ils se livreraient en s'abandonnant aux sonorités et rythmes de cette musique, que le jazz vient d'un ailleurs mais qui se trouve d'abord être un « en bas ».

Venu d'en bas ? C'est bien le cas du trompettiste Tim Tooney qui a fait ses apprentissages de la vie et de la musique dans les faubourgs pauvres, populeux et crasseux de Southampton. C'est aussi le cas du pianiste Novecento qui, nourrisson, fut découvert par un soutier afro-américain du Virginian, langé et couché dans une boîte en carton sur le piano à queue de la salle de bal des premières classes, sans doute abandonné là par un couple d'émigrants des ponts inférieurs dans un geste de désespoir, de fatigue ou d'impuissance - fruit de leur noire misère. Le soutier prend alors sur lui d'adopter puis d'élever l'enfant dans la salle des machines, parmi des sacs et des pelletées de charbon aussi noir que sa peau. Devenu adolescent, Novecento retrouve le piano du grand salon et, durant des nuits entières, apprend seul à en jouer, se mettant à jongler, comme une vedette de cirque (ses acrobaties par temps de roulis ne nous sont pas épargnées), avec les rythmes, mélodies et harmonies au point de devenir plus tard une attraction pour le gratin du navire et le pivot de son orchestre de danses.

11 Ce sont non seulement des mondes à part, des univers en négatif qui sont ici esquissés, mais des mondes de parodie autant que de corruption évoquant aussi bien les lieux originaires du jazz plus ou moins légendaires (les quartiers chauds, souvent violents, mélangés et interlopes de La Nouvelle-Orléans) que la capacité qu'a cette musique d'inverser les signes de la présence sonore et de la présentation de soi (du noir au blanc, et du blanc au noir, prolongeant en cela les danses et chants blackface ainsi que les pantomimes des minstrels ${ }^{7}$ ), et où le corps de l'instrumentiste se déracialise, se fait autre (Ellison $1964: 234$ ). Nulle place pour on ne sait quel essentialisme : le jazz n'est pas qu'une affaire de peau et de couleur, non plus que de communauté ou de culture (y avait-il, pouvait-il même y avoir une culture noire authentique dans le sud des États-Unis au début $\mathrm{du} \mathrm{xx}^{\mathrm{e}}$ siècle ?). Ce sont les musiciens qui donnent traits, grains et couleurs à la musique. En plus d'être antiphonique (le couple appel/réponse dont l'origine remonte aux gospel songs), le jazz était stéréophonique avant même que cette technique de reproduction du son ne fût inventée. Et c'est sur cette spatialisation du sonore, sur cette dislocation de la tonalité, de la mesure, du rythme, du doigté, du pas de danse, que se fonde et se nourrit ce qu'il conviendrait d'appeler sa geste musicale (au sens ancien de "geste", c'est-à-dire 
« exploit »). Il se manifeste là une esthétique, si ce n'est même une philosophie du décalage.

Cette manière d'être dans la musique et de faire de la musique se serait en quelque sorte développée au cours du long métissage culturel (la diaspora noire) et de l'extrême dramatisation sociale (l'esclavage, le racisme, la ségrégation) dont le jazz est issu. Ce qui tint lieu de tradition ne put être qu'une invention permanente de la tradition, c'est-à-dire un dépassement voire un rejet de tout ce qui faisait système, doctrine, dogme ou folklore, alors perçu comme un dispositif non seulement de contrainte mais d'oppression. Transformer des cantiques religieux (gospel songs), des chants de travail (work songs), des mélopées de forçats (chain-gang songs), des complaintes (blues) en objets de distraction et de plaisir relève moins de la provocation, de la protestation, que de l'ironie, c'est-à-dire de la distanciation. Non pas "lieux de mémoire » mais tableaux ou portraits acoustiques souvent rythmiques. «Il existe», remarque William Lhamon (2004: 85), «des pans entiers de la culture qui n'exhibent pas de racines et ne produisent pas de modèles élaborés dans les monuments culturels. » Ce fut le cas du jazz. De là, sans doute, l'un de ses traits pour ainsi dire génériques d'apparaître constamment « sur le gril », d'affirmer une identité, fût-elle une identité "yoyo" (comme le déclare le compagnon de Novecento), et de renvoyer à cette « double conscience " qu'a conceptualisée W.E.B. Du Bois (1964) au début du siècle. C'est-à-dire le sentiment d'être noir et américain dans une Amérique blanche, esclavagiste et raciste - sujet et objet d'une tension non réconciliée, voire non conciliable, qui projette l'identité afro-américaine hors du temps historique, lequel s'apparente plutôt au temps d'un parcours, d'une traversée, à une modalité de passage d'un lieu ou d'un milieu à un autre. Le fait est que l'histoire du jazz, par exemple, s'inscrit surtout et avant tout dans une géographie d'abord rurale, puis urbaine, parfois suburbaine ou underground: routes, fleuves, voies ferrées, rivages, villes, quartiers, rues, boîtes et caves ont non seulement inspiré nombre de compositions jazzistiques mais servi à désigner des périodes, des centres de gravité esthétiques ou des styles musicaux comme les styles New Orleans, Saint Louis, Kansas City, Chicago, West Coast, New York, Minton's, etc. ${ }^{8}$ Par ailleurs, au niveau de la technique musicale, Franck Bergerot (1996:58-66) a bien mis en évidence la dimension essentiellement spatiale que revêt l'improvisation en jazz : l'exploration de la grille harmonique, l'art du voicing (placement des accords sans recourir à la fondamentale), la production de " nappes de sons ", récemment l'usage de la modalité renvoient plus à une plastique qu'à une rhétorique sonore, à l'image d'une sculpture ou d'un tableau.

13 Novecento symbolise de manière frappante non seulement le surgissement de l'altérité $d u$ jazz et dans le jazz - une altérité tout autant native que rétive (Novecento envoie tout « au cul», autrement dit "aux chiottes », lois, règlements, terre, gratte-ciel, guerre..., et même, nous l'avons vu, l'invention du jazz en la personne de Jelly Roll Morton) - mais aussi son nomadisme, son extraterritorialité: sa vie durant, le pianiste Novecento refusera de mettre le pied à terre et passera son temps en allers-retours sur l'océan, n'ayant pour toute racine que le tabouret d'un piano qui roule par gros temps. Il périra assis sur une caisse bourrée d'explosifs, indifférent mais entêté, dans les cales du Virginian qui, transformé en navire-hôpital et désarmé après la Seconde Guerre mondiale, était voué à la destruction par dynamitage.

Bien qu'il eût souvent exprimé le désir d'observer comment, de la terre, la mer se donnait à voir, Novecento ne fut jamais capable d'aller au-delà des trois premières marches de la passerelle lorsque le navire se trouvait à quai. Non parce qu'on ne sait quel mal de terre 
l'aurait soudain pris de nausées, ou qu'il fût paralysé par ce qu'il voyait surgir devant lui, mais parce qu'il ne pouvait voir où ceci - la terre, les rues, les gratte-ciel, les passants, les femmes - commençait ni où cela menait ni même où cela finissait : c'était comme « un clavier de millions, de milliards de touches» (Baricco 1999: 72). Au moins les coursives, salles et cales du paquebot étaient-elles à portée de vue; au moins les quatre-vingt-huit touches du piano étaient-elles à portée de main et permettaient-elles d'en tirer, en soi et pour soi, malgré l'échelle graduée des leviers en noir et blanc, malgré la suite imposée des noires et des blanches, quelque chose d'illimité qu'on était seul à savoir inventer et à pouvoir maitriser : un air de musique qui, avec de la finitude, crée de l'infinitude - ce qu'ont très bien compris les musiciens de jazz dans leurs rapports, parfois politiquement et culturellement revendiqués et affirmés (Jones 1997 ; Carles et Comolli 2000 ; Pierrepont 2002), avec la variation et l'improvisation. Là encore, tout se passe comme si la « musique du diable» (Mosley 1997) telle que l'avait incarnée mythiquement Jelly Roll Morton pouvait venir d'un corps blanc, non par contagion mais du fait d'une commune humaine condition, du sentiment partagé de la précarité de l'existence: le déracinement, le dépaysement et l'identité en l'occurrence flottante. Il est intéressant de noter que, dans son maître ouvrage, Paul Gilroy (2003: 19) s'appuie précisément sur l'image du navire comme «symbole-pivot et point de départ» de son analyse de la culture diasporique : "Système, écrit Gilroy, en mouvement, micropolitique, microcultrurel et vivant » (ibid.). «Les navires, ajoute-t-il plus loin, attirent immédiatement l'attention sur le "passage du milieu", sur la circulation des idées et des activistes ainsi que sur les déplacements d'objets culturels et politiques fondamentaux : tracts, livres, disques et chœurs. " Mais faut-il ajouter que les navires furent aussi, pendant quelques siècles, des navires négriers par quoi se déplacèrent et périrent des corps, des langages et des cosmogonies, en un mot : des cultures?

Un certain goût du paradoxe nous conduirait à avancer que Novecento - bien que blanc et, peut-être, parce que blanc - personnifie ce qu'est le jazz au fond: une musique toute faite de zébrage, de tangage, de collage, de bricolage, de brassage, de marronage, une irruption sans cesse reprise et réactualisée de l'autre dans le même, du noir dans le blanc et du blanc dans le noir, du mineur dans le majeur et inversement, telles ces touches de piano desquelles Novecento ne pourra plus jamais se détacher. C'est donc dans le même qu'en jazz se fabrique de l'autre. Et c'est cette sorte d'« exotisme intime " que, par une autobiographisation du son (Jamin et Williams 2001: 331), le jazz fait surgir et vient mettre en notes, à l'image de ce surgissement de l'altérité intime à quoi conduit toute entreprise autobiographique et sa mise en mots, comme l'a superbement prouvé et éprouvé Michel Leiris qui, lui-même, avait vu très tôt dans cette musique - ce dès le début des années 1920 - non seulement une façon de se dépayser (par l'écoute, par la danse) mais une expression même du nouveau rapport au monde qu'implique notre modernité (Leiris 1973 : 161-162), c'est-à-dire l'avènement du transitoire et du fugitif dont parlait déjà Baudelaire à propos de la mode, et que Jean-Paul Sartre (1970:681), après la Seconde Guerre mondiale, retiendra lui aussi du jazz : une musique qui s'adresse à une autre part de nous-mêmes, à « la meilleure, à la plus sèche, à la plus libre, à celle qui ne veut ni mélodie ni ritournelle, mais l'éclat assourdissant de l'instant». Le jazz aurait donc la faculté de révéler, de convoquer, d'imposer non seulement cette " altérité essentielle ou intime» dont parle Marc Augé (1992: 29), mais une forme d'altérité vocale, musicale, esthétique qui se traduirait par une accumulation de composantes qui s'additionnent sans se détruire, si bien qu'on est toujours et qu'on n'est plus jamais « chez soi » (ibid. : 136) ${ }^{9}$. 


\section{«Le plus bel air peut-être que l'Amérique nous ait envoyé »}

16 C'est à une autre œuvre littéraire que Novecento que nous nous référerons afin de poursuivre l'examen de cette irruption du jeu du même et de l'autre, et du même dans l'autre tel qu'il peut se donner à voir et se faire entendre dans la simple évocation d'un vieux standard de jazz, où les signes de sa reconnaissance, les règles de son exécution, la position de ses interprètes semblent biaisés ou, bien plus, semblent s'inverser. On y retrouve cette faculté « de combiner, de joindre, de partager, d'attirer les contraires et de jouer sur les oppositions » qui est non seulement propre au jazz mais qui, selon William Lhamon (2004: 4), caractérise la culture diasporique du Nouveau Monde, sa modernité, son étrangeté et, à travers les songs de la Tin Pan Alley (voir infra), toutes ses «petites tragédies instantanées ». Lorsqu'il écrit La Nausée en 1936, Jean-Paul Sartre l'avait déjà pressenti au point d'en user comme d'un indicatif narratif ou d'une anaphore, laissant courir au fil des pages l'aiguille d'un gramophone sur le disque usé d'une chanson, presque une rengaine.

17 Cette chanson, ce standard, c'est Some of These Days. Un standard sans doute (Williams 2006) mais aussi, d'abord et dans tous les sens du terme, un tube. On rappellera en effet que l'emploi de ce dernier mot pour désigner un air à succès renvoie métonymiquement aux cylindres de phonographes par lesquels la musique enregistrée accéda pour la première fois à une reproductibilité industrielle. Préférer le mot français au terme qui s'est imposé dans le monde du jazz et au-delà offre d'abord l'avantage de renvoyer au support sur lequel Sophie Tucker, la créatrice de la chanson, l'a pour la première fois enregistrée, à New York, le 24 février 1911 : un cylindre Amberol de 4 minutes gravé pour Edison Records sous le numéro 4M-691. Mais c'est surtout insister sur le succès immense, inédit, qu'a rencontré en Europe et singulièrement en France cet air de la Tin Pan Alley ${ }^{10}$. De la commotion produite par cette chanson sur les sensibilités européennes, les remarques et souvenirs du poète belge Robert Goffin témoignent éloquemment. Auteur d'un des premiers ouvrages critiques consacrés au jazz à proprement parler, observateur irremplaçable de ce que Jean Cocteau appelait « l'arrivée américaine du rythme », Goffin (1932 : 47) analyse, depuis son observatoire bruxellois, la diffusion des airs américains :

«[...] dès 1912 et 1913 et surtout au début de la guerre, le jazz existe et se répand dans tous les États. Les airs se multiplient et courent les routes; les chanteurs populaires assiègent tous les coins de rue; des compagnies à plusieurs voix parcourent les campagnes, soutenues parfois par un grêle banjo, parfois par l'un ou l'autre instrument de cuivre qui fait rire les populations curieuses. Les premières chansons de jazz, lourdes de nostalgie et de banalité, avec des airs très mélodiques, où le contretemps nègre n'est encore qu'une douce et sinueuse inflexion qui se modifiera bientôt, font leur véritable apparition: "Poney boy", "Are you from Dixie", "Lily in the Valley", chanson bientôt traduite en français et monnayée par les orchestrions de 1914 sous le titre de "Poupée d'amour"; et puis surtout le sublime et extraordinaire "Some of These Days", sanglot prophétique qui traversa les continents et fit frémir tous les cœurs ouverts au mystère, le plus bel air peut-être que l'Amérique nous ait envoyé, créé bien avant la guerre pour les voix surhumaines et célestes de Sophie Tucher [sic], de Louis Armstrong ou de Cab Calloways [resic]. »

Poète, Goffin est un lyrique épris de subjectivité. Son histoire du jazz est absolument personnelle. On modulera donc son jugement à la lumière de celui d'un individu réputé moins enthousiaste. À partir de 1926, la substitution de l'enregistrement électrique à 
l'enregistrement acoustique décide Henry Prunières, fondateur et directeur de la prestigieuse Revue musicale, à assurer dans cette dernière une rubrique «La musique par disques». Ce musicien et musicologue distingué le fait sans le moindre ostracisme puisqu'en février 1928, après avoir commenté un nouvel enregistrement de Pelléas et Mélisande par Piero Coppola, quelques belles faces de Ninon Vallin, «incomparable soprano lyrique », ou du "grand artiste » Fritz Kreisler, il achève sa chronique par une courte rubrique « Jazz et chanson » que voici (Prunières $1928: 86$ ) :

« Sophie Tucker a une voix prodigieuse, une voix de feu, d'une ardeur étrange, cri sauvage qui semble venir du fond des âges, jaillir de la forêt vierge. On n'a pas oublié son interprétation de Some of These Days avec le jazz de Ted Lewis chez Columbia, un des plus beaux disques que je connaisse ; elle vient de donner à Odéon un disque intéressant: Blue River, dont le motif nostalgique est indéfiniment répété et There's a Craddle in Carolina. J'avoue qu'après cette voix démoniaque l'art si sobre et si délicieux de Raquel Meller dans Nena semble un peu fade. Hungria a plus d'accent et de vigueur rythmique (Odéon). »

19 Commentons pour l'heure ce texte d'une simple remarque: que quelqu'un qui fait profession d'en écouter et de les écouter en musicien considère Some of These Days comme «le plus beau disque [qu'il] connaisse» valide l'émotion de Goffin. D'autant que Prunières, écrivant ces lignes, n'abdique pas tout esprit critique puisqu'il qualifie Blue River de disque seulement "intéressant ». Un an plus tard, dans le cours d'une rubrique «La musique par disques » particulièrement développée, on relève les lignes suivantes (Prunières 1929 : 274-275) : "Sophie Tucker chante : I Know that My Baby is Cheatin'on Me, pour Columbia. C'est intéressant comme tout ce que fait cette artiste extraordinaire, mais ne saurait approcher de créations antérieures comme Some of These Days ou [1]e prodigieux Virginia [...]. » La convergence des expertises, fort contrastées, de Goffin et de Prunières établit qu'à la fin des années 1920, en Europe, Sophie Tucker est considérée comme une interprète de premier plan et que Some of These Days est devenu un étalon à l'aune duquel, dans l'ordre de la musique populaire, toute réussite semble devoir être mesurée.

C'est donc dire qu'en plaçant en $1938^{11}$ cette chanson au cœur du dispositif romanesque de La Nausée, Sartre, loin d'être un pionnier, se signale comme le dernier venu ou presque d'une série d'écrivains qui, dès les années 1920, ont fait de ce morceau un objet de littérature. Tout en souhaitant rendre la page de Sartre, qui a souvent été commentée, à un contexte, à un cotexte qui ont été jusqu'ici négligés, c'est pourtant par La Nausée que nous allons continuer cette étude en noir, blanc et blond de Some of These Days. Pour deux ordres de raisons. Le premier tient au fait que si cette chanson a survécu dans les mémoires après la Seconde Guerre mondiale, c'est au roman de Sartre qu'elle le doit. L'impressionnante reconnaissance critique dont ont bénéficié ce song et son interprète en France à la fin des Années folles rend en effet par contraste plus spectaculaire l'oubli dans lequel Sophie Tucker et son répertoire entrent dès le début des années 1930 : absents des scènes, fussent-elles mentales, intimes ; absents bientôt des plateaux de phono, de pickup; n'a-t-il pas fallu attendre la fin $\mathrm{du} \mathrm{xx}^{\mathrm{e}}$ siècle pour que la plus connue des interprétations de Some of These Days par Sophie Tucker devienne à nouveau disponible commercialement sous une forme autre que le 78 tours original? Pendant plus d'un demi-siècle, Some of These Days n'aura donc vécu que d'une existence muette, existence seulement textuelle, purement littéraire - le motif sartrien par excellence - qui, s'agissant du « plus bel air peut-être que l'Amérique nous ait envoyé », fait figure de paradoxe. 
21 Mais il y a, à continuer notre étude par Sartre, un motif plus décisif encore que l'espèce d'obligation d'hommage à laquelle invitent les considérations ci-dessus exposées. C'est que l'auteur de La Nausée, dans les quelques pages qu'il consacre à la chanson, accumule spectaculairement les bévues. Loin qu'il s'agisse ici pour nous de moquer les gaffes réelles ou supposées de Sartre, nous montrerons au contraire que cette série d'erreurs - mais qu'est-ce au juste qu'une "erreur " dans une fiction narrative? -, s'organisant en système de signification, nous conduit précisément au cœur des questions de marronnage, de déracialisation et d'altérité que la lecture de Novecento : pianiste a déjà permis de mettre en place.

\section{Une musique blanche inventée par un Noir}

Dans La Nausée, donc, on se rappelle que le narrateur, Antoine Roquentin, pris soudain de nostalgie au souvenir de son amie Anny dont il est séparé, se fait jouer par la serveuse, sur le phonographe du bistrot «Au rendez-vous des cheminots» qu'il fréquente régulièrement, une chanson qui le touche particulièrement: "Madeleine, jouez-moi un air, au phono, vous serez gentille. Celui qui me plait, vous savez, Some of These Days. » (Sartre 1938 : 37) Dès la première occurrence de cet air, c'est-à-dire à la page 37 de l'édition blanche, le lecteur aborde ce qu'à la suite de John Searle (1982: 116-118) on pourrait nommer un "archipel référentiel ». L'énoncé se déroule sur un mode factuel, s'inscrit dans une dimension historique et, en l'occurrence, dans ce qui est de l'ordre du récit de vie, et ceci sans grand dommage narratif: le genre journal rehaussé par des intertitres empruntés au semainier s'y prête, voire y invite (Sartre 1938 : 38) :

«Vendredi [...] 5 heures et demie.

Madeleine tourne la manivelle du phonographe. Pourvu qu'elle ne se soit pas trompée, qu'elle n'ait pas mis, comme l'autre jour, le grand air de Cavalleria Rusticana. Mais non, c'est bien ça, je reconnais l'air dès les premières mesures. C'est un vieux rag-time avec refrain chanté. Je l'ai entendu siffler en 1917 par des soldats américains dans les rues de La Rochelle. Il doit dater d'avant-guerre. Mais l'enregistrement est beaucoup plus récent. Tout de même, c'est le plus vieux disque de la collection, un disque Pathé pour aiguille à saphir. »

Pourtant, sans qu'il n'y paraisse, dès cette première mention de Some of These Days, le « réel » menace déjà de n'exister plus qu'en termes d' « effet ». La première difficulté est occasionnée par la définition de Some of These Days comme étant un rag-time ${ }^{12}$. C'est du moins ce que Joë Bousquet semble penser, qui, en mai 1938, quelques semaines après la parution de La Nausée, adresse à Sartre une lettre dont la réponse du philosophe nous permet d'inférer le contenu ${ }^{13}$ :

«Je suis un peu confus d'avoir laissé une faute comme celle que vous me signalez et, s'il y avait un autre tirage de La Nausée, je la ferai disparaître. Mais je crois que Some of These Days, qui date d'avant-guerre, était un ragtime. En tout cas, il figure dans le pot-pourri de ragtimes de Jack Hylton ${ }^{14}$. »

Sur quoi Bousquet se fonde-t-il pour reprocher à Sartre la «faute » qui consiste à faire de Some of These Days un ragtime? Sur la structure du thème? Si, avec ses 32 mesures organisées en $\mathrm{ABCD}$, Some of These Days présente une "formule moins fréquente " (Baudoin 1994b: 1163) que le traditionnel AABA qui définit la majorité des standards, cette structure non circulaire se retrouve couramment parmi les premiers ragtimes publiés (Sutton 2003 : xi). Soulignons d'ailleurs, avec Allan Sutton (ibid., notre traduction), que «les débats pour déterminer ce qui relève ou non du ragtime peuvent être 
extrêmement complexes et bien souvent oiseux ». Ragtime ou non, il reste que Some of These Days s'apparente à un de ces airs « ringards " produits dans la Tin Pan Alley et dont parle Gunther Schuller (1998: 142), autrement dit la « rue des casseroles » ou l'« allée des scies » - artère comprise entre la 5e et la 6e Avenue de New York où furent composés presque à la chaîne, selon un mécanisme de taylorisation de la culture dite de masse, airs et chansons populaires qui allaient faire les beaux jours de la comédie musicale américaine et constituer une des bases du répertoire des musiciens de jazz (Williams 2006). Peut-être Bousquet dénie-t-il le statut de ragtime à Some of These Days au nom de ce qu'il s'agit d'une chanson (Fréchet $2001: 178$ ). Aussi bien est-ce parce qu'il n'a pas «été originellement conçu comme une pièce instrumentale plutôt que vocale » que Sutton (2003: xi) peut aujourd'hui décider d'écarter tel ou tel morceau de sa récente et très complète discographie du ragtime. Et il est vrai que la précision de Roquentin (« C'est un vieux rag-time avec refrain chanté ») semble envisager cette question du chant comme déterminant le genre. Vrai encore, comme on va le voir, que Some of These Days a été d'emblée conçu par son auteur comme un song.

Mais Sartre n'a pas tort de défendre malgré tout, face à Joë Bousquet, l'hypothèse de cette appartenance générique - ou plutôt stylistique. Au moment où Some of These Days est composé, le jazz n'existe pas : ni le mot ni la chose, et c'est par le terme de ragtime que, jusqu'à la Première Guerre mondiale au moins, les musiciens de La Nouvelle-Orléans désignent leur musique - les souvenirs de Sidney Bechet ou de Jelly Roll Morton ${ }^{15}$ en témoignent. La musique que Roquentin entend "siffler en 1917 par des soldats américains dans les rues de La Rochelle " peut donc recevoir ce nom et il est d'ailleurs probable que Sartre transpose, dans l'allusion de Roquentin, une expérience musicale qui lui est propre ${ }^{16}$. De fait, en novembre 1917, le jeune Sartre accompagne sa mère et son beau-père, Joseph Mancy, qui, venant de se marier, se sont installés dans cette ville; il entre alors en classe de 4e A (latin, grec) au lycée de garçons. Or, en cette même année, les ports atlantiques français accueillent les régiments américains venus secourir les Alliés. C'est ainsi que le 15th Regimental Band débarque à Brest le 1er janvier 1918. À sa tête, le chef James Reese Europe (1881-1919), roi du ragtime new-yorkais. Alors que le régiment stationne à Saint-Nazaire, sa clique donne, à Nantes, un concert de marches françaises et américaines qui, avec The Memphis Blues, s'achève en triomphale démonstration de ragtime (Badger 1995 : 167). À en croire Noble Sissle (1889-1975), alors tambour-major de la formation, qui adresse son témoignage au Post Dispatch de Saint Louis, c'est là, à cet instant, que le virus du ragtime est inoculé à une France exsangue qui avait bien besoin de ce cordial : "Colonel Hayward [le commandant en chef du régiment] has brought his band over here and started ragtimitis in France !» (Ibid. ; voir également Southern 1976 : 287-288)

Passons à la deuxième «faute » sartrienne. Car le passage que nous analysons est de fait embarrassé, en particulier sur le plan des temporalités ${ }^{17}$, mais aussi - ils finissent par se confondre - sur un plan que l'on dira technologique. Le syntagme « un disque Pathé pour aiguille à saphir » dont use Roquentin est en effet proprement dépourvu de sens, étant donné qu'un disque des années 1920 est soit à aiguille, soit à saphir. Ces deux standards technologiques sont mutuellement incompatibles, l'un relevant d'une gravure latérale lisible, par une aiguille, sur les flancs du sillon (c'est le classique 78 tours), l'autre d'une gravure verticale lisible, dans le fond du sillon, par une petite bille de verre inusable appelée saphir. Jusqu'en 1926, un disque Pathé est forcément à saphir (Pathé ne se lance dans la production des 78 tours que l'année suivante), c'est-à-dire qu'il use d'une 
technologie volontiers perçue comme archaïque et si vieillotte effectivement que la firme en programme l'abandon en 1928, ne produisant plus, par la suite, que des disques à aiguille. Admettons cependant que l'expression contradictoire employée par Sartre vise un disque à gravure verticale : la consultation des catalogues Pathé montre qu'il n'existe pas d'interprétation féminine de Some of These Days sur disque à saphir. Ni sous ce titre ni sous celui de Bébé d'amour, choisi par Francis Salabert pour publier, en 1913, la version française de la chanson de Shelton Brooks.

Où l'on commence à concevoir que l'accumulation des précisions dans cette page qui, de toutes les manières, proclame sa vérité, pourrait bien renvoyer à des problèmes de technique romanesque. Plutôt que de prononcer trop vite la dislocation de l'archipel référentiel, compliquons encore un peu plus les choses et admettons que le roman, ici et comme toujours, avance en mettant en jeu, en travaillant des données techniques, sociales, des réminiscences de toutes sortes, fragments en eux-mêmes parfaitement authentiques mais de la distorsion, de l'appropriation desquels vit la littérature. Notre découverte d'une face jusqu'ici passée inaperçue rend en effet plus clair le travail de l'écrivain dans cette page de La Nausée. Il s'agit d'un morceau enregistré à Paris au début d'octobre 1918 par le 158th U.S. Infantry Band que dirige le lieutenant Etzweiler. Ce thème, signé Billy Montgomery et publié en 1917, a pour titre - pas de quoi alerter un sartrien - The Story Book Ball. Il s'agit d'un disque Pathé, disque à saphir donc, qui procure à l'auditeur, au terme de sa première minute, la surprise d'entendre s'élever une mélodie autre que celle qu'annonce le titre de la face: le refrain de Some of These Days. Si les musiciens de James Reese Europe sont noirs, le 158th réunit quant à lui des soldats blancs. Ces deux fanfares interprètent pourtant les succès de la Tin Pan Alley sur un mode binaire et avec les syncopes caractéristiques du ragtime (Southern 1976: 287 etc.). Roulements des tambours, choc des woodblocks, éclats de fifres... En dépit de certains jazz effects, l'auditeur de The Story Book Ball se retrouve plus proche infiniment de ce qu'un chef d'orchestre blanc comme John Philip Sousa (1854-1932) a pu faire découvrir des marches américaines aux visiteurs de l'Exposition universelle de $1900^{18}$ que de ce qui va très bientôt se faire entendre sous le nom de jazz. Et l'on voit que Some of These Days doit finalement bel et bien quelque chose aux disques Pathé et au style ragtime - même si c'est en biais. Et que Sartre en conséquence ne fantasme pas la scène du phonographe de $L a$ Nausée à partir de rien.

28 Cependant, la version exigée par Roquentin n'est ni celle de Sophie Tucker en 1911 (elle n'a jamais existé sous forme de disque) ni celle (tronquée et purement instrumentale) du lieutenant Etzweiler et de ses hommes. Il ne s'agit pas non plus de la rare et passionnante version de la firme Victor en 1929 : passée inaperçue en France, elle n'y a probablement jamais été diffusée. Dans l'évocation de La Nausée (Sartre 1938: 39-40), Roquentin se réfère, selon toute apparence, à l'interprétation de 1926. Et c'est là que l'énoncé achève, avec un suspense tout rhétorique, de basculer dans la fiction pure et simple, c'est-à-dire dans l'assertion feinte (Searle $1982: 110$; Schaeffer 1999: 133 et suiv.) :

«Tout à l'heure viendra le refrain: c'est lui surtout que j'aime et la manière abrupte dont il se jette en avant, comme une falaise contre la mer. Pour l'instant, c'est le jazz qui joue ; il n'y a pas de mélodie, juste des notes, une myriade de petites secousses. Elles ne connaissent pas de repos, un ordre inflexible les fait naître, et les détruit, sans leur laisser jamais le loisir de se reprendre, d'exister pour soi. Elles courent, elles se pressent, elles me frappent au passage d'un coup sec et s'anéantissent. J'aimerais bien les retenir, mais je sais que, si j'arrivais à en arrêter une, il ne resterait plus entre mes doigts qu'un son canaille et languissant [...]. Quelques secondes encore et la Négresse va chanter. Ça semble inévitable, si forte 
est la nécessité de cette musique : rien ne peut l'interrompre, rien qui vienne de ce temps où le monde est affalé ; elle cessera d'elle-même, par ordre. Si j'aime cette belle voix, c'est surtout pour ça : ce n'est ni pour son ampleur ni pour sa tristesse, c'est qu'elle est l'événement que tant de notes ont préparé, de si loin, en mourant pour qu'il naisse. »

Some of These Days, You'll miss me honey... est donc chanté, écrit Sartre et nous dit Roquentin, par une Négresse, une voix à l'aride pureté, hors du temps, hors de l'existence, hors du monde. À la manière de la petite phrase de la Sonate de Vinteuil chez Proust, ce « ragtime » va jouer tout au long de La Nausée le rôle d'un leitmotiv indiquant la distance, la partance, l'errance, mais aussi un au-delà de la propre vie du narrateur. En l'écoutant, Roquentin ne peut s'empêcher de penser à ces autres existences tout aussi contingentes que la sienne mais celles-ci délivrées de l'angoisse d'exister: le compositeur et la chanteuse, qui sont peut-être déjà morts mais qui s'imposent à soi comme quelque chose de précieux et d'à moitié légendaire, comme des existants invisibles que l'on peut, à l'envi, faire revivre à travers les sillons usés et rayés d'un disque. Telle est la "morale» (négative) de Novecento et le remède de Roquentin pour échapper à la nausée que lui procure son existence « en trop "... Une chanson, la musique, n'existe pas à la façon des choses non plus qu'à celle des corps : il est « impossible de la toucher » même si elle nous touche, nous prend, nous charme ou nous taillade (Cook 2006:76); mais ce n'est pas sous la forme de morceau qu'elle nous atteint ; l'expression, et donc la métaphore - « morceau de musique » - trouve là ses limites. La musique est insécable : «[...] Si je me levais, se dit Roquentin, si j'arrachais ce disque du plateau qui le supporte et si je le cassais en deux, je ne l'atteindrais pas, elle.» (Sartre 1938: 238) Où l'on retrouve les propos d'Alain (1963: 116) sur les chants populaires : «[...] la musique contraste toujours avec ce que la chanson dit; mais elle contraste toujours comme il faut, opposant à la misère humaine une consolation d'avance et comme une vue sur un long déroulement du temps. »

À son départ de Bouville, à la fin du récit, Roquentin se fait jouer une fois encore sur le phono Some of These Days; il imagine alors le compositeur écrivant le thème et les paroles quelque part dans un gratte-ciel ensoleillé de New York (Sartre $1938: 240)$ :

«Il est assis, en bras de chemise, devant son piano ; il a un goût de fumée dans la bouche et, vaguement, un fantôme d'air dans la tête. Some of These Days. Tom va venir dans une heure avec sa gourde plate sur la fesse ; alors ils s'affaleront tous deux dans les fauteuils de cuir et ils boiront de grandes rasades d'alcool et le feu du ciel viendra flamber leurs gorges, ils sentiront le poids d'un immense soleil torride. Mais d'abord il faut noter cet air. Some of These Days. La main moite saisit le crayon sur le piano. "Some of These Days, You'll miss me honey." Ça s'est passé comme ça. Comme ça ou autrement, mais peu importe. C'est comme ça qu'elle est née. C'est le corps usé de ce juif aux sourcils de charbon qu'elle a choisi pour naître. [...] »

31 Là encore, Sartre a tout faux dans cette évocation qui se veut pourtant factuelle, référentielle, réaliste. D'une part, cette chanson fut écrite et composée en 1910 par Shelton Brooks, un pianiste noir américain (1886-1975), et non par un Juif new-yorkais " aux sourcils de charbon » comme l'imagine Roquentin. D'autre part, la chanteuse qui popularisa Some of These Days à la fin des années 1910 n'est pas une « Négresse » mais une Blanche, Sophie Tucker, qui en fit même son air de prédilection, sa trademark, au point d'en emprunter le titre pour son autobiographie (Tucker 1945). 


\section{Blackface}

Juive d'origine russe ${ }^{19}$, Sonia Kalish (1884-1966) est née «on the road» (comme Novecento naquit « on the ocean »), dans une ferme entre la Russie et la mer Baltique ; sa mère, émigrante, n'avait que 17 ans à sa naissance, et c'était déjà son deuxième enfant (elle en eut quatre). Quelques semaines plus tard, elle fut nommée Sonya ou Sophie Abuza, du nom que son père, Charles, avait emprunté à un compagnon italien rencontré lors de sa fuite de la Russie tsariste et qui, insoumis comme lui ( $A W O L »$, écrit Sophie Tucker ${ }^{20}$ ), était mort sur le bateau de l'exil; il l'avait déclaré comme étant le sien à l'officier d'immigration des États-Unis, avant de s'installer à Boston, puis à Hartford (Connecticut) pour y ouvrir un restaurant kasher. Mariée en 1903 à Louis Tuck (d'où elle fit dériver son nom de scène), Sophie Tucker connut d'abord le succès comme blackface et coon-shouter, engagée dans une troupe de minstrels de 1905 à 1910²1. Un an avant sa mort, en 1965, face aux questions pressantes d'une de ses petites-nièces, Lois Young-Tulin, qui avait noué avec elle de forts liens depuis le milieu des années 1950 et qui, devenue militante des droits civiques, s'étonnait qu'en tant que fille d'émigrants juifs elle eût participé à ces comédies de vaudeville aux fortes résonances racistes, Sophie Tucker tenta de se justifier en ces termes (Young-Tulin 2001 : 152, notre traduction) :

« Pour des Juifs comme Eddie Cantor, Al Jolson ou moi (j'étais l'une des premières femmes de scène à revêtir la panoplie du blackface), il était important de montrer que nous étions de vrais Américains, même si nous étions considérés comme des artistes ethniques, c'est-à-dire juifs. De ce point de vue, le coon-singing présentait bien des atouts. Avec les Afro-Américains qui étaient la cible de notre humour, le vaudeville tel que nous le pratiquions démontrait que notre culture d'origine étrangère, à nous immigrants, devait être prise en compte tout comme devait l'être celle des Afro-Américains pourtant jugée comme inférieure. Le blackface incarnait aussi une souffrance liée à l'immigration et au déracinement, expérience en cela analogue à celle des Afro-Américains. »

Cette attitude défensive, au raisonnement quelque peu sophistique, ne doit cependant pas masquer la vérité musicologique et sociologique (nous y reviendrons plus loin) de ce que laisse entendre ici Sophie Tucker : les traits harmoniques (inflexion tonale, diatonisme), rythmiques (syncope, contretemps), mélodiques et vocaux (timbre guttural, registre aigu, émission ductile, scansion voyellisée), instrumentaux (détimbrage, sourdine, glissando) et gestuels (pas de danse tour à tour feutrés et sautillants) que, sous une forme parodique, le blackface et le coon-singing empruntèrent aux expressions et traditions musicales des AfroAméricains, avaient en quelque sorte ouvert la voie, au même titre que le ragtime et le cake walk, à la réception du jazz au sein de la société blanche américaine ainsi qu'à son exploitation par l'industrie culturelle naissante. Que cela se fît sous l'angle de la farce, voire de la moquerie ou de la satire, et fût mis en scène par des immigrants, en l'occurrence juifs pour la plupart - ils avaient au moins en commun avec les Noirs de n'être plus d'aucune terre, d'aucun lieu, d'aucune origine si ce n'est celle de l'entre-deux, tel Novecento -, n'ôta rien à leur impact sur les mentalités et les sensibilités. Bien au contraire : ces déconcertants « ménestrels " préparèrent le terrain émotionnel pour que la musique noire fût non seulement reconnue mais réactivée et régénérée par ceux-là mêmes qui avaient été, et continuaient d'être, au mieux l'objet de pastiches et parodies, au pire les victimes d'exclusion et de persécution. L'humour, le sourire, le rire pouvait avoir raison du mépris et du rejet, et l'excentricité, raison aussi des convenances, assurances et préjugés de race, de classe ou même de sexe (Levet 2002). En ce sens, les 
spectacles des minstrels et blackfaces, loin d'être l'expression d'un racisme épidermique, ce faisant simpliste, comme le note William Lhamon $(2004: 7,48,263)$, proposaient des lieux et schèmes de représentation du mélange et du métissage, une grammaire vocale et gestuelle d'une identité fluide, toujours en cours de formation : « Quand j'ôtais mes gants, confie Sophie Tucker, et montrais que j'étais blanche, il y avait dans le public comme un soupir auquel succédait un énorme éclat de rire. Alors, j'introduisais dans mes chansons quelques mots en yiddish non seulement pour faire un pied de nez aux auditeurs mais pour leur faire comprendre qui j'étais vraiment. » (Young-Tulin 2001 : 132) On verra plus loin ce qu'il lui en coûta de jouer ainsi, sur scène et dans ses chansons, de et avec ses origines. En somme, comme le remarque Riv-Ellen Prell (1998 : 136-137) :

"Juifs et Africains-Américains étaient en train de créer quelque chose d'entièrement nouveau: une culture urbaine, jazzy et subversive. Ils chamboulèrent les codes de bienséance, de maintien et de contrôle du plaisir qu'avait institués l'esprit victorien et puritain au tournant $d u x^{e}$ siècle. Ne seraitce que par leur manière effrontée de se présenter, de jouer et de chanter sur scène, des artistes comme Sophie Tucker prouvèrent que les femmes avaient des "appétits" et étaient capables de jouer un rôle dans l'espace public. Elles pouvaient chanter ce qu'elles étaient, ce qu'elles désiraient, et dire leur volonté de n'être dirigées par aucun homme. »

Ce n'est donc pas la dimension raciste du blackface qui amena Sophie Tucker à abandonner ce genre de spectacle (nous avons vu que, face aux reproches de sa petitenièce, elle la dénia). C'est plutôt parce qu'elle s'y sentit très vite à l'étroit, le grimage, la mascarade l'empêchant d'être comme elle aurait souhaité paraître sur scène, c'est-à-dire «avec le minois, les atours et les désirs d'une femme »: «Le blackface, soulignait-elle, m'ôtait ma féminité. » (Young-Tulin $2001: 132$ )

Un moment à l'affiche des Ziegfeld Follies, Sophie Tucker connut ensuite une carrière indépendante et une réussite "à l'américaine » comme chanteuse et actrice de revues, de comédies musicales, dont la célèbre Louisania Lou, ou de films musicaux. Au milieu des années 1920, elle forma un orchestre de cinq musiciens (violon, saxophone, clarinette, piano, batterie), The Five Kings of Syncopation, et s'autoproclama " The Queen of Jazz » (à l'instar de Paul Whiteman, qui se nomma «The King of Jazz », d'Al Jolson, «The Jazz Singer », ou du compositeur Irving Berlin, qui se dit « Mr Jazz Himself », tous blancs et/ou juifs [Gabbard 2002: 4 et infra]). Devenue, avec le temps, The Last of the Red-hot Mamas, en raison non seulement du titre de la pièce de Jack Yellen qu'elle inclut dans son répertoire mais des sujets à forte connotation sexuelle de la plupart de ses chansons, en raison également de sa blondeur, de sa voix tonitruante et de sa corpulence - son amie Ada Smith Ducongé (Bricktop 1983: 165-166), qui fut une des premières tenancières de bar américain à Paris, installée rue Pigalle après la Première Guerre mondiale (Leiris 1992 : 97-99), l'avait surnommée « 3 B », c'est-à-dire : «Blond, Big and Booming» -, Sophie Tucker connut une renommée qui dépassa les limites de Broadway et de la Tin Pan Alley.

\section{Voix noire, corps blanc (et vice-versa)}

Selon un jugement exprimé dans l'autobiographie de la grande chanteuse de jazz noire Ethel Waters (1896-1977), Sophie Tucker fut la «dernière représentante de l'époque héroïque [celle des minstrels], elle poussait sa voix, à la nègre, comme on disait alors [c'est-à-dire une voix de gorge projetée] - spécialité qui a disparu depuis » (Waters 1952 : 
193). Milton Mezz Mezzrow (1966: 81) parlait, quant à lui, plus incisif et persifleur, de « minables contrefaçons de la chose authentique ».

À la croisée d'un imaginaire et de données culturelles voire physiologiques, la question de la voix noire, de la voix noire de Sophie Tucker, en particulier, crûment posée par l'équivoque sartrienne, a beaucoup et de longue date fait écrire. Peut-on être blanche et chanter comme une Noire? Comme une Noire s'entend: avec une voix noire ; un grain, un timbre, un son noirs? Question qui trouve son symétrique : si, à partir de Leontine Price (née en 1927), la question du chanteur classique de peau noire a commencé à ne plus en être une, il n'en est pas allé de même pour les artistes de la génération antérieure. Il y avait un scandale à entendre un Roland Hayes (1887-1976), une Florence Cole-Talbert (1890-1961), voire une Marian Anderson (1897-1993) interpréter autre chose que des negro-spirituals: chanter Lakmé, Haendel ou Pagliacci. Un scandale à entendre un chanteur noir usant des techniques du chant savant occidental pour atteindre à cette pureté d'émission qui est la fin ultime visée par cette tradition - un chanteur noir à la voix blanche, en somme. Sophie Tucker et Roland Hayes, depuis leurs positions symétriques, dans la mesure même où ils mettent à mal des représentations esthétiques et politiques très profondément installées dans les esprits, sont des embrayeurs du lapsus et du fantasme ${ }^{22}$.

Dès les années 1930, le journaliste italien Angelo Nizza, bon connaisseur d'un jazz qu'il était allé découvrir dans sa patrie américaine, renversant l'ordre de préséance indiqué par Ethel Waters et Mezz Mezzrow, se lança dans une quête des origines qui n'a pas cessé depuis $^{23}$ et mit tout simplement les chanteuses noires à l'école de Sophie Tucker. Le 24 février 1934 - on est à quelques mois de l'invasion de l'Abyssinie par l'Italie mussolinienne -, il salue dans La Stampa le passage d'Armstrong dans la péninsule par un long texte intitulé «Le Voci del jazz. Armstrong in Italia». On y lit les remarques suivantes (Nizza $2004:$ 405, notre traduction) :

«[Cab] Calloway a dans le cœur, dans le sang, ce besoin atavique de se donner tout entier à son art singulier. Ses éblouissantes pyrotechnies vocales, sa voix de gorge portée dans l'aigu font de lui le plus moderne et le plus paradoxal des chanteurs qu'on ait jamais entendus. Sa manière, qui se distingue de celle d'Armstrong et qui est imitée par beaucoup de chanteurs de couleur des deux sexes, a pour initiatrice une Blanche, une israélite d'origine allemande [sic], née dans le Connecticut [resic], Sophie Tucker. Cette dame, désormais âgée, chante, de sa voix de contralto ardente et lumineuse, de vieux airs de jazz depuis 1906. Elle se présentait alors devant le public américain avec les traditionnels "ragtimes" et d'autres chansons populaires. On doit à Sophie Tucker une première exceptionnelle : avoir porté à sa perfection l'art du chant syncopé et l'avoir révélé même aux Nègres [di aver rivelato agli stessi negri e di aver affinato definitivamente il modo di cantare ritmando e controritmando con la voce]. »

Si faire de Sophie Tucker l'inventeur du chant syncopé, pour ne pas dire la mère du chant noir, est abusif, on a vu que Juifs et Noirs se côtoient dès avant 1900 dans le monde du vaudeville (Mezzrow 1966 : 80-81). Et, se côtoyant, s'écoutent. On doit à Mark Berresford et John R.T. Davies la réédition en disque compact de la précieuse version de 1929 de Some of These Days par Sophie Tucker, la seule dans laquelle la chanteuse semble avoir été accompagnée par un orchestre noir. Voyons dans quels termes Berresford justifie sa présence dans un disque entièrement consacré aux chanteuses noires ${ }^{24}$ (Berresford 2000 : non pag., notre traduction) : 
40 Linclusion de Sophie Tucker dans ce disque compact surprendra peut-être certains auditeurs. Il est cependant indéniable que son style déclamatoire et sa diction sous haute énergie [her powerhouse delivery] ont influencé bien des chanteurs de blues, et que Tucker en retour a subi l'influence de nombre d'interprètes noirs. [...] Son influence sur la première génération des chanteuses de blues à avoir enregistré est absolument patente [à l'écoute du morceau] et on ne peut que regretter qu'elle n'ait pas davantage travaillé avec des musiciens noirs. "

$41 \mathrm{Au}$ vu de tels jugements, on conçoit que les écrivains français, d'ailleurs longtemps tributaires du seul disque pour se figurer son interprète, ont, à leurs errances, quelques circonstances atténuantes. Les écrivains français - car la «faute » à laquelle, si l'on peut dire, Sartre a donné sa forme classique, n'est-elle pas déjà présente dans les lignes de Prunières que nous avons citées plus haut? Lorsqu'il évoque la « voix prodigieuse » de Sophie Tucker, « une voix de feu, d'une ardeur étrange, cri sauvage qui semble venir du fond des âges, jaillir de la forêt vierge ", le critique nous entraine au cœur de l'Afrique, à mille lieues assurément tant de Broadway que de la steppe...

42

De même, Robert Desnos, lorsqu'il compare les registre et timbre de voix du chanteur et chef d'orchestre noir américain Noble Sissle (1889-1975) avec ceux de Sophie Tucker. Le " seul chanteur ", écrit-il dans Le Soir du 13 septembre 1928, " que l'on puisse placer sur le même plan [qu'elle], le seul qui chante "nègre" avec cette passion pathétique qui donne à la voix des hommes de couleur plus que du charme et de la séduction, mais de l'empire sur ceux qui les écoutent» (Desnos 1987: 156). On s'étonne d'abord de voir Desnos associer le ténor léger de Sissle, beaucoup plus proche de l'art d'un Roland Hayes que de celui d'un Armstrong, à l'organe énergique et voilé de Sophie Tucker. Il est vrai que Desnos, en rapportant le caractère "nègre» du chant à une certaine qualité du pathétique, en préférant en somme le terrain de l'interprétation à celui de la voix, ouvre ainsi à Sophie Tucker la possibilité d'être considérée effectivement comme une chanteuse noire... D'où la surprise de voir quelques mois plus tard ce même Desnos, qui reconnait comme noir le chant de Sissle, dénier ce statut à celui de Paul Robeson. Le 26 avril 1929, sa chronique de disques pour Le Merle, après une allusion à « Sophie Tucker dont l'éloge n'est plus à faire, tant son nom est lié à l'évolution de l'art phonographique ", s'achève en effet par les lignes suivantes : « Signalons enfin le plus beau disque de Robeson enregistré chez Gramophone : Sonny Boy, où le chanteur anglais se plie, non sans un excès de grâces civilisées, au rythme mélancolique des chants noirs. » (Desnos 1987 : 164) La voix noire, le chant noir décidément sont un piège - piège auquel Jean Cocteau, dernier venu de la théorie des auteurs-auditeurs des années 1920 et 1930 à avoir rêvé à propos du cas Sophie Tucker, ne se laisse pas prendre. Dans un texte ambigu intitulé « Danger secret du rythme noir » et publié à Pâques 1943 dans le mensuel La Légion, l'auteur des Enfants terribles corrige en quelque sorte Sartre et, proposant une généalogie moins surprenante que celle établie par Nizza, note : « [...] de célèbres chanteuses blanches imitent les Négresses. Par exemple Sophie Tucker " (Cocteau $2004:$ 279). Mais il est vrai qu'à cette époque Cocteau est devenu, en ce qui concerne le jazz, l'élève de Hugues Panassié ${ }^{25}$ et qu'il écrit en un temps où la popularité du trio Louis Armstrong-Bessie Smith-Paul Robeson a fixé les quelques valeurs définissant subjectivement la voix noire - valeurs qui, nous semble-t-il, lui sont aujourd'hui encore associées : un timbre sinon toujours rocailleux du moins voilé d'une part; d'autre part une inclination au registre grave, à la voix de basse. 


\section{Yiddishe Momme}

43 Alors que ses disques enchantent les amoureux du phono et que sa voix fait rêver les critiques, Sophie Tucker entame, au début des années 1930, une tournée triomphale en Europe. Celle-ci s'acheva toutefois sur un four au théâtre de l'Empire à Paris où, accompagnée par un orchestre de jazz anonyme, elle donna une série de concerts en février 1931. Lors de l'un d'entre eux, un mercredi soir, le public se déchaîna, lui infligeant une véritable bronca. Ce ne fut pas à cause de son interprétation de Some of These Days dont elle avait pris la peine de faire traduire et de chanter le refrain en français ${ }^{26}$, et qui fut vivement acclamé, mais à cause de la chanson My Yiddishe Momme sur laquelle elle termina, ce soir-là et pour la première fois, son tour de chant à l'Empire - chanson qui, composée en 1925 par Jack Yellen et Lou Pollack et enregistrée en 1926, avait remporté un grand succès commercial outre-Manche et outre-Atlantique. Face à un public qu'elle pensait acquis, elle la chanta, comme sur le disque, d'abord en anglais puis en yiddish. Mais à peine eut-elle prononcé les première paroles dans cette langue que des huées et sifflets lancés par des voyous du poulailler s'emparèrent d'une grande partie du public, à quoi tentèrent de s'opposer les ovations de l'autre, bien moins nombreuses : « Je me trouvai dans un sale pétrin, constate-t-elle dans son autobiographie, et je me sentis démunie face à ces gens qui finirent par s'empoigner dans les travées - ce que je n'avais jamais vu se produire dans une salle de spectacle.» (Tucker $1945: 257$, notre traduction) Elle eut beau interrompre la chanson et enchainer, non sans esprit d'à-propos, avec Happy Days Are Here Again ${ }^{27}$, elle dut quitter la scène pour éviter l'émeute (ibid. : 258). De ce côtéci de l'Atlantique, n'est plus de mise l'éclat de rire qu'évoquait Sophie Tucker lorsque, abandonnant sa panoplie de blackface, elle révélait au public anglo-américain ses véritables origines. Le surlendemain, la presse américaine titra: Sophie Tucker Hissed off Paris Stage (ibid. : 258). Et ce fut la première et la dernière fois que Sophie Tucker se produisit sur la scène d'un théâtre parisien. Dépitée, elle fit part de cet échec à Ada Smith, le mettant sur le compte de la vague d'antisémitisme qui semblait de nouveau envahir la France et dont elle devint ainsi une des premières vedettes étrangères du music-hall à être victime ${ }^{28}$ (Bricktop 1983 : 165).

L'épisode est, de fait, éloquent et étonnant, d'autant plus que, selon Léon Poliakov (1981: 459), l'antisémitisme français connaît, dans ces années-là (1925-1930), son « étiage le plus bas ». La séduction, l'enthousiasme, l'ovation que venait de déclencher Some of These Days, sans doute à cause de ce qui, dans cette chanson, évoquait musicalement l'exotisme, si ce n'est le "primitivisme ${ }^{29}$ ", mais qui demeurait factice, quelque peu caricatural à la manière des anciens minstrels, furent de très courte durée. La langue aux accents germaniques, les inflexions slaves de l'accompagnement et la déclamation en forme de sprechegesang de My Yiddishe Momme renvoyaient à un autre type d'altérité, celui-ci sans strass ni carton-pâte ni "négrification", qui, pour certains, apparaissait maudit tout autant que menaçant et ne pouvait avoir voix sinon au chapitre du moins au divertissement... Une Blanche pouvait « se la chanter à la nègre ", mais l'épisode parisien démontra qu'elle ne pouvait, ici, sur scène - c'est-à-dire en représentation - se montrer et s'exhiber comme juive. Ce n'était plus d'un exotisme de sons, de rythmes et de couleurs qu'il s'agissait - le Blanc ne peut plus affirmer sa « splendeur » (Poliakov 1981: 425) par la seule contre-image du Noir qu'il s'autorise ou se plait à contrefaire à travers la voix ou par le grimage : d'autres Blancs rôdent en lui et chez lui (Sartre 2005: 124 et suiv.) - mais 
de ce qu'il conviendrait d'appeler un " exotisme de l'intérieur ", c'est-à-dire un exotisme « double » au sens de double jeu, voire de duplicité. Sophie Tucker semblait donc incarner les propos bien connus, et terribles, du critique littéraire Henry Mencken à l'encontre des Juifs : «Ils pensent en yiddish et ils écrivent en anglais!» (in Poliakov 1981: 425), puisqu'elle mettait les deux langues sur un même plan, chantant donc en yiddish et en anglais une chanson qui renvoyait ouvertement à ses origines, aux deux faces d'un même disque, au recto-verso de sa propre identité.

Le four parisien, désolant, n'empêcha pas les écrivains français de continuer un temps à célébrer une chanteuse dont la voix donnait à Desnos le sentiment d'être " perdu sur une côte par une nuit de tempête » (Desnos 1987 : 151). La chanteuse ou sa chanson. En 1939, à la veille de la déclaration de guerre, c'est encore ce même Some of These Days, alors qualifié de blues, qu'évoque Michel Leiris dans le premier tome de La Règle du jeu, Biffures (1948: 258, 271). En août de cette même année, il l'entend diffuser par un haut-parleur sur le paquebot qui l'emmène en Grèce où il va en vacances (comme Roquentin il vient de se séparer d'une amante) : «[...] Je partis pour la Grèce, écrit-il, et entendis le blues Some of These Days, grands coups de trompe me taillant des à-pics en plein cœur... » Mais est-ce bien la version de Sophie Tucker qu'entend Leiris ce jour-là? Son évocation et son émotion ("blues", "grands coups de trompe ", " à-pics ») - rapportées, il est vrai, de façon laconique - feraient plutôt penser à l'interprétation qu'en donna Louis Armstrong en 1929, et où se met en place ce qui allait caractériser le schéma formel de son style : solo de trompette, vocal, break du saxophone ou de la clarinette, et chorus de trompette final dans lequel Armstrong élargit sa tessiture dans l'aigu, allant même jusqu'au contre-ré ${ }^{30}$ (Schuller 1997 : 142).

\section{Le tiers inclus}

Dans son autobiographie, Sophie Tucker (1945: 114, notre traduction) relate ainsi sa rencontre avec Shelton Brooks et sa découverte de Some of These Days à la fin de 1910, à Chicago, où elle se trouve dans une suite d'hôtel, après une représentation de la comédie musicale Louisiana Lou $^{31}$ :

« Un jour Mollie [la camériste] entra et se tint face à moi, les mains sur les hanches mais avec un tel regard que je la crus en proie à une crise de démence: "Eh bien jeune dame, me lança-t-elle, vous croyez-vous donc devenue tellement importante que vous n'êtes même plus capable d'écouter une chanson écrite par un compositeur de couleur? Il y a ici ce jeune gars Shelton Brooks [il a 25 ans] qui traîne et attend, la langue pendant comme un chien, que vous prêtiez une oreille à la chanson qu'il vient d'écrire. Au lieu de cela, vous vous agitez dans tous les sens à battre des ailes comme un poulet à qui on a coupé la tête. Vous ne pouvez faire autrement que de recevoir ce charmant garçon qui vaut bien plus qu'une réponse évasive. - Bon, d'accord! lui promis-je, j'écouterai sa chanson. Tu peux le lui dire. Vous pouvez le lui dire vous-même !" me répondit-elle, sur quoi elle le fit entrer. Au moment où j'entendis Some of These Days, on aurait pu me frapper à devenir sourde que je ne pouvais rien oublier de la chanson. Une chanson comme ça! Elle avait tout pour elle. Est-il besoin de le prouver ? Je l'ai chantée pendant trente ans et j'en ai fait mon indicatif. Je l'ai retournée dans tous les sens, je l'ai interprétée de toutes les façons imaginables, comme une chanson réaliste, comme un drame, comme une fantaisie, comme une romance, chaque fois les gens l'ont aimée et la redemandaient. Some of These Days est une de ces grandes chansons dont on se rappelle et que l'on peut chanter et qu'on chantera des années et des années encore, comme certaines des chansons de Stephen Foster ${ }^{32}$. » 
Antoine Roquentin, et partant Jean-Paul Sartre, inversent par conséquent les signes des binômes Blanc/Noir, Juif/Nègre, comme ils intervertissent, à l'intérieur de ceux-ci, les attributions homme/femme : la qualité de juif, dans la réalité possédée par une femme (Sophie Tucker), est assignée au niveau de la narration à un homme, et la couleur de la peau et le genre, objectivement noire et masculin (Shelton Brooks), deviennent blanche et féminin dans le récit. L'erreur est certes troublante, d'autant plus que Sartre se disait amateur de jazz, mais, tout compte fait, elle ne constitue pas une hérésie puisque les rapports d'opposition et de tension sont préservés. La chanson Some of These Days se présente bien, que ce soit dans la réalité ou dans la fiction, comme une fusion d'altérités, une combinaison d'éléments distincts et, en l'occurrence, disparates - comme la résultante d'une sorte de mixage de genres, de peaux, de mots, de sons. Tout au plus pourrait-on dire que cette ultime erreur de Sartre constitue, nous l'avons suggéré, une feintise, c'est-à-dire une licence par rapport à la référentialité, mais qui n'altère nullement sa véridicité. Il en propose simplement un autre modèle, une autre combinaison, laquelle - nous le verrons - n'est pas sans liens avec un autre type de référentialité, celle-là, musicologique. Reste qu'il est étrange que Sartre persiste dans l'erreur alors que dans une nouvelle du Mur, L'Enfance d'un chef, publiée en janvier 1939, mais qu'il a achevé d'écrire en juillet 1938 (La Nausée est parue en avril 1938, au moment donc de la rédaction de L'Enfance d'un chef: Sartre aurait pu corriger cette faute sur épreuves), le héros, Lucien, découvre et, mélancolique, écoute les voix reproduites sur un gramophone de «Sophie Tucker et d'Al Johnston » (qu'il faut lire évidemment Jolson). Or Sartre, qui a vu un autre film d'Al Jolson, The Singing Fool, réalisé par Lloyd Bacon en 1928 et sorti en France en 1930 sous le titre Le Fou chantant (Beauvoir 1960: 60), sait pertinemment bien qu'il est blanc de peau et a joué des rôles de blackface tout comme Sophie Tucker (elle et lui sont d'ailleurs, nous l'avons vu, d'origine juive et russe). Le fait qu'ils soient tous deux - et eux seuls - musicalement associés dans ce syntagme narratif de L'Enfance d'un chef (Sartre 1979 : 187) laisse encore plus ouverte la question de savoir si ce n'est pas sciemment que, pour des raisons fictionnelles, donc dramatiques, Sartre transmue dans La Nausée Sophie Tucker en Négresse, comme pour mieux faire sentir et rappeler, ainsi que le dirait Denis Hollier (1982 : 206), l'absence que la chanson Some of These Days chante : non seulement celle de l'amante de Roquentin mais celle-là même de la chanteuse, et au-delà - ce qui est la thématique de La Nausée - celle de l'être, qui se trouve dès lors et presque au sens propre néantisé... Être juif, blanc ou noir n'est pas une question d'essence. On le sait depuis le poème du fantaisiste Georges Fourest (publié en 1909), des Négresses peuvent être blondes!

Ce n'est donc pas l'enregistrement sur cylindre de Some of These Days par Sophie Tucker ${ }^{33}$ que Roquentin commente dans son journal. Ce n'est pas non plus l'interprétation de 1929 - la plus proche, pourtant, du moment d'élaboration de La Nausée - mais la version Columbia de 1926. On achèvera de s'en convaincre en écoutant le morceau en parallèle avec le commentaire, finalement très précis, qu'en produit le roman (Sartre $1938: 39$ )原:

«Tout à l'heure viendra le refrain: c'est lui surtout que j'aime et la manière abrupte dont il se jette en avant, comme une falaise dans la mer. Pour l'instant c'est le jazz qui joue. Il n'y a pas de mélodie, juste des notes, une myriade de petites secousses ».

Ce « jazz », c'est celui de Ted Lewis. De Lewis, nous dirons simplement que sa popularité a été aussi grande hier qu'est aujourd'hui profond le mépris dans lequel le tiennent les histoires du jazz. Il est exemplairement une des victimes du purisme, par ailleurs plein 
d'effets positifs, de Hugues Panassié (1934 : 317), et l'impatience d'un Roquentin qui ne vit que dans l'attente de l'irruption de la voix de « la Négresse " (Quelques secondes encore et la Négresse va chanter » [Sartre 1938 : 39]) n'est au fond que la transposition littéraire du dédain panassiéen. Ce qui est dommage. Car s'il avait prêté attention à l'orchestre et à son chef, Sartre-Roquentin eût pu, sans risquer la confusion, rêver sur le rôle des Juifs dans l'invention de la musique populaire américaine : Ted Lewis fait en effet partie de la liste des vedettes juives de la scène que - on l'a vu - dresse Mezz Mezzrow.

Theodore Leopold Friedman, né à Circleville, dans l'Ohio, en 1890, succède au milieu des années 1920 comme vedette de la maison Columbia et prodigieux vendeur de disques à un autre clarinettiste, noir celui-là: Wilbur Sweatman. L'un comme l'autre pratiquent le style de clarinette excentrique qui fut une spécialité appréciée au tournant de 1900 et que George McClennon, Fess Williams, Wilton Crawley ou Boyd Senter - tous noirs, sauf le dernier - ont illustré au long des années 1910 et 1920. Une clarinette qui gémit, sanglote, parle, s'esclaffe sans cesse. Cette clarinette qui n'a ni la sinuosité melliflue d'un Jimmy Noone, d'un Barney Bigard, d'un Benny Goodman, ni la rudesse inspirée d'un Sidney Bechet, d'un Johnny Dodds ou d'un Leon Rappolo est un pur produit des spectacles de vaudeville (Wilbur Sweatman, cinquante ans avant Roland Kirk, aime à jouer de trois instruments à la fois), de blackface (McClennon noirçit au bouchon brûlé son visage, pourtant noir, jusque dans les années 1930). Elle tombe du même coup dans un angle mort de l'histoire des spectacles, pour ne rien dire de l'histoire du jazz. Comme le signale Joel B. O'Sickey ${ }^{34}$, Ted Lewis se situe à la croisée de la gas-pipe clarinet illustrée par les grands vaudevillistes noirs et de la clarinette klezmer - sa joie panique, son angoisse virtuose et glapissante - inventée dans les années 1920 par un Naftule Brandwein, un Dave Tarras, l'une et l'autre usant des mêmes effets expressionnistes. Ted Lewis figure en lui-même ce carrefour que le présent article s'essaie à explorer : méprisé par des jazzmen comme Mezz Mezzrow ou Eddie Condon ${ }^{35}$, ses contemporains, il est pourtant capable, notamment pour sa tournée européenne de 1929, de s'entourer des meilleurs instrumentistes (blancs) disponibles : George Brunies, Muggsy Spanier, Jimmy Dorsey, etc. C'est à l'occasion de ces concerts parisiens de 1928 et 1929 que Robert Desnos célébrera avec un fol enthousiasme les talents de musicien, de showman mais aussi et surtout de chanteur - de diseur, en fait - de celui que l'on appelait le tragédien en chapeau haut de forme (Desnos 1987 : 167-168).

51 C'est donc au sein de l'orchestre ou à sa tête, mais non derrière le piano du compositeur, que Roquentin aurait pu (dû) entendre un Juif. Pour l'instant, s'appuyant sur le beau jeu de basse à vent d'Harry Barth, c'est en effet, durant 24 mesures, «le jazz qui joue ». Et qui joue straight, mené par une trompette et le violon aigrelet de Sol Shapiro qui détaillent à l'unisson la mélodie. Après un «bref silence» (Sartre 1938: 40), de fait parfaitement perceptible sur le disque, la Négresse blonde chante enfin :

Some of these days

You'll miss me honey,

Some of these days

You're gonna be so lonely.

You'll miss my hugging,

You're gonna miss my kissing.

You're gonna miss me, honey,

When I'm far away.

I feel so lonely for you only,

Cause you know, honey,

You've always had your way. 
And when you leave me,

You know it's gonna grieve me.

Gonna miss your big fat mamma,

[your mamma

Some of these days. la voix rude de la chanteuse. À force d'écouter et de réécouter ce morceau, d'abord par devoir, bientôt par plaisir, à force de suivre la belle ligne de guitare de Maurice Aten, d'apprécier comment la voix étire savamment les syllabes, il se produit de fait le sentiment d'une nécessité. On finit par ne plus pouvoir imaginer le morceau autrement. Ne plus pouvoir l'imaginer sans cette clarinette (et non un «saxophone» [Sartre 1938 : 238] : « quatre notes de saxophone [...] qui vont et viennent » comme l'écrit un Roquentin ici victime d'un Sartre qui, d'évidence, restitue le contenu du disque de mémoire), une clarinette qui gazouille et répand ses intervalles surprenants, ses roulades vieillottes et charmantes au-dessus de la voix de Sophie Tucker. Ainsi en va-t-il en tout cas pour Roquentin, dont la nausée reflue, "si forte est la nécessité de cette musique » (Sartre 1938 : 238). Sur un accord de sol majeur, la note, ré majeur - « so... me of these days »-, si vibrée qu'elle tend au shake et que redouble Ted Lewis à la clarinette, Sophie Tucker (Armstrong, au sein de son Hot Five, n'en use pas autrement) rameute l'orchestre pour le chorus final, puis se tait, laissant la place aux instruments : «Le disque s'est arrêté.» (Sartre $1938: 42$ )

Le jeu auquel nous nous sommes livrés, qui consiste donc à évaluer le degré de référentialité de Some of These Days, n'a au fond que peu d'importance au regard de l'économie même du récit de La Nausée. Que Roquentin entende un disque à saphir ou à aiguille, une voix noire à la place d'une voix blanche, qu'il imagine un Juif en train de composer sur son piano à la place d'un pianiste noir, cela n'empêche nullement que les effets de la chanson sur le narrateur seront les mêmes. Il reste que si Alessandro Barrico fait du noir avec du blanc, Jean-Paul Sartre faisait donc du blanc avec du noir. Mais, dans les deux cas, c'est sur ce pouvoir de transformation, de "transfiguration » du jazz, écrirait André Schaeffner $(1988,2002)$, que l'accent est mis, c'est-à-dire sur cette capacité d'assimiler le folklore, voire tous les folklores, de les transcender et même de les réinventer musicalement. De ce point de vue, Sartre allait plus loin que Barrico puisqu'il intégra dans l'idiome musical du jazz, et dans sa représentation littéraire, une composante juive sur laquelle certains musiciens et écrivains européens ont très tôt insisté.

\section{Sophie, Dinah, Sarah, Irving, Isaac et les autres}

Dans cet ordre d'idée, nul n'a précédé le chef d'orchestre suisse Ernest Ansermet (1984: 33) et son article séminal de 1919, publié dans la Revue romande à la suite d'une prestation à Londres du Southern Syncopated Orchestra de Will Marion Cook. Ansermet y mettait en valeur, outre le génie d'un jeune Noir nommé Sidney Bechet, la dimension instantanée du jazz, dimension par laquelle le rôle du compositeur se voit minoré :

« Tous les caractères de cet art, en effet, montrent en lui un type parfait de ce qu'on appelle l'art populaire - l'art qui est encore à sa période de tradition orale. Il est bien indifférent, après cela, que la musique nègre soit écrite par des Juifs russes, des Juifs allemands, ou quelque Anglo-Saxon corrompu. » 
Liée que la musique populaire américaine est le fruit d'une collaboration entre diverses communautés au premier rang desquelles se distinguent la noire et la juive est, en France, presque aussi ancienne que cette musique même (Schaeffner 1988 : 106). En 1943, encore, dans un texte qui, il est vrai, fait explicitement référence à Ansermet, ne voit-on pas Cocteau souligner, après « la secousse du premier jazz, [...] les échanges, les demi-sang, le métissage des musiques. Des Juifs russes empoignaient à New York les syncopes nègres, $\mathrm{y}$ joignaient leur mélancolie et c'est de cette rencontre que naquirent les premiers grands rag-times que nous déchiffrait Doucet au "Bœuf sur le toit" "? (Cocteau 2000 : 278-279).

Dis longtemps, il est vrai, un compositeur comme Irving Berlin (Israel Baline de son nom d'état civil, 1888-1989) est bien connu en France. Originaire, comme Sophie Tucker, de Russie, ce musicien autodidacte venu aux États-Unis en 1893 avec ses parents pour fuir les pogroms, a produit à la chaîne, tout au long de son siècle d'existence, des centaines de mélodie pour la Tin Pan Alley. Dès les années 1910, Pathé-France en enregistre certaines sur disques et cylindres, par exemple et sous divers titres (Alexandre, Le Célèbre Pas de l'ours...) son premier grand succès: Alexander's Ragtime Band. C'est aussi l'époque où le revuiste Jacques-Charles, qui joua un rôle majeur mais ignoré dans «l'arrivée américaine du rythme ", fait sa connaissance à New York et découvre, à ses côtés, la vie nocturne de la ville (Jacques-Charles 1958 : 175-177). Autant que son talent de mélodiste, on admire l'art qu'il a eu de donner à son œuvre une dimension industrielle. Ainsi Pierre Mac Orlan, remarquable connaisseur du disque et des musiques populaires, se prend-il, en 1924, à rêver (Mac Orlan 1925 : 193) :

"Quand on apprend, au hasard d'une conversation compétente, que le compositeur Irving Berlin, dont le nom, pour cette raison, devient encore plus mystérieux, gagne un million par an, on ne peut s'empêcher de le situer à la tête d'une étonnante usine musicale, distribuant le fox-trot, les blues et les shimmy, en disques plats comme des crêpes de blés noirs. »

On le voit, la figure du compositeur juif d'airs populaires américains doit être comprise comme étant devenue en France, dès le début des années 1920, un véritable mythe. Ce mythe, Mac Orlan lui donnera, longtemps avant Sartre, sa première forme proprement littéraire sous l'apparence d'une courte nouvelle qu'il fit paraître en 1927 dans le quotidien Paris Matinal. La nouvelle est fondée sur le montage parallèle de deux destins sans contacts imaginables, que les hasards de la vie et les nécessités de l'histoire vont amener à se croiser.

Dans sa première version, la nouvelle - un conte, plutôt - a pour titre Banana Oil et l'héroïne, qui est chanteuse, a nom Dinah. Noire, cette héroïne habite sur les rives du Mississippi. Elle «n'a jamais que 25 ans, mais grâce à la tradition populaire des chansons de sa race, elle est plus vieille que la vieille maison décrépie qu'elle habite avec ses dix frères et sœurs » (Mac Orlan 1986: 52). Car elle tient son art de son aïeule esclave, qui «avait appris des chansons d'une douceur anormale, d'une grande douceur, comme limitée par la crainte » (ibid.). Dinah regarde les steamboats parcourir le fleuve et écoute les « interminables chœurs, montés par demi-tons » (ibid. : 51) qui s'en échappent.

Lorsqu'il republie son conte, quelques mois après sa première parution, Mac Orlan y introduit des modifications. L'héroïne prend le nom de Sarah et le conte, le nom de l'héroïne. On peut se demander pourquoi. Banana Oil est le titre d'un succès des années 1920 - chanson comique que la chanteuse américaine Vaughn de Leath (l'une des trois voix féminines qui, avec Lee Morse et bien sûr Sophie Tucker, enchantent le poète Desnos [1987 : 151]) a popularisée bien au-delà des États-Unis. Quant à Dinah, premier prénom de 
l'héroïne, c'est, on le sait, une chanson créée par Ethel Waters en 1925 dans la revue Plantation et rapidement confiée au disque avec succès. Par son interprétation de 1926, Joséphine Baker, «l'étoile noire des Folies Bergères ", ainsi que la désigne l'étiquette du disque Odéon sur lequel elle l'a enregistrée, achève de populariser la chanson en France. Peut-être les résonances comiques de Banana Oil ont-elles tout compte fait paru embarrassantes à Mac Orlan pour servir d'enseigne à un récit feutré et émouvant. Sans doute le nom de Dinah s'est-il trouvé finalement encombré de trop de références, trop fortes, trop actuelles ${ }^{36}$. Il reste que, dans ce jeu de substitution, la chanteuse noire du conte se voit attribuer le nom même de la matriarche du peuple juif, la mère d'Isaac. Ce qui a pour avantage de préparer le lecteur à faire la connaissance de l'autre personnage de la fiction. Le texte (ibid. : 53), construit sur une série d'oppositions qui se résoudront finalement en un accord, pivote en effet nettement autour d'un blanc typographique qui fait axe de symétrie :

«En regardant passer le grand bateau de plaisance qui promenait les espoirs de son peuple, en écoutant les chœurs traditionnels qui se mêlaient à l'attendrissante pureté du ciel américain, Sarah rentrait son linge chauffé par le soleil. Elle écoutait en souriant le coassement des grenouilles de la Louisiane et les gémissements bien modulés d'un bébé noir qui pleurait consciencieusement à l'ombre d'une vieille niche à chien.

Isaac Hambourg, de son côté, mais très loin de cette image, escalada l'échelle du paquebot mouillé par la pluie et par la brume salée et se trouva transi et dépaysé sur le pont des émigrants encombré de bagages. [...] Des Galiciennes, des Polonaises en robes fleuries, des Juives très désolées, formaient autour de son maigre bagage un groupe confus qui brouillait le paysage des quais et les hautes grues électriques qui laissaient l'eau du ciel s'égoutter au bout de leurs longs bras. »

Voilà de retour les navires si bien analysés par Paul Gilroy (2003), mis en récit par Alessandro Baricco (1999) et, probablement, devenus lieu d'inspiration pour un Darius Milhaud (2005) qui y formula la combinatoire musicale transatlantique d'une nouvelle «création du monde »... On se doute qu'Isaac Hambourg ne possède pour tout bien qu' u un violon enfermé dans un tout petit cercueil doublé de peluche rouge » (ibid.). Il est musicien. «Sa mémoire était riche d'un passé autoritaire, parfois tragique, mais fleuri de chansons gracieuses que sa grand-mère lui avait chantées ${ }^{37}$. " (Ibid. : 53-54) Arrivé à New York, il vit d'abord misérablement puis remonte la pente " presque insensiblement » (ibid . : 54). Histoire d'une assimilation (ibid. : 54-55) :

« La nuit, Isaac Hambourg jouait du violon ou du banjo dans un orchestre fameux, à l'ombre d'un hélicon formidable. Autour de lui, les belles jambes des filles de New York obéissaient à l'élan sentimental du jazz. Peu à peu, tout en s'épongeant le front entre chaque fox-trot applaudi, Isaac prit l'habitude sociale du smoking. Quand il fut à point grâce aux lumières de l'hôtel, à la présence des femmes de la nuit, à quelques réminiscences de l'Europe centrale, il échangea son nom contre celui de Ted Nicholson. Il connut alors la saveur des boissons fraîches et antihygiéniques et assez de loisir pour mettre en ordre ses souvenirs et s'inspirer de sa misère ancienne [...]. Ted rentra chez lui, un soir, dans son "bloc" avec la connaissance lumineuse qu'il pourrait vendre cette mélancolie héritée en lui donnant le rythme qu'il avait subi, en évitant les autos et en charmant les belles de son époque.

C'est ainsi qu'il écrivit, pour commencer, une chanson fameuse chantée par cette même Sarah qui découvrit sa voix, une belle chanson chaude et pathétique comme Some of These Days, si vous voulez.

Cette chanson, écrite à la gloire de Sarah, pénètre aujourd'hui partout et s'épanouit irrésistiblement dans la pièce où le phonographe la révèle comme une invasion de sentiments populaires, rares et délicats. » 
61 On le voit, dans ce texte écrit l'année même où la France se prend de passion pour le disque de Sophie Tucker et Ted Lewis, Mac Orlan brasse, avec dix ans d'avance, exactement le même matériel historique, sociologique, géographique, musicologique, politique, exactement les mêmes stéréotypes que Sartre. Dans Sarah comme dans La Nausée, le compositeur est juif et la chanteuse noire. Mais comment se fait-il que cette identique répartition des rôles ne soit pas, cette fois, synonyme d'erreur? On pourrait penser que Mac Orlan, tout comme Desnos critique professionnel de disques, est mieux informé que Sartre. Ainsi son Isaac Hambourg-Ted Nicholson est-il très consciemment un mixte - au moins l'onomastique le suggère-t-elle avec insistance - d'Irving Berlin et de Ted Lewis. L'hypothèse de l'information est pourtant en l'occurrence sans pertinence : longtemps avant Sartre et même avant Prunières et Desnos, Mac Orlan était tombé - et avec quel éclat ! -, dans le piège d'une Sophie Tucker « chanteuse de couleur ». Sarah n'est en effet que l'expansion, la mise en fiction de certaines de ses chroniques de disques de l'année 1927. Dans ces dernières, l'auteur de Quai des brumes prononce un éloge de Some of These Days auprès duquel même le lyrisme des Goffin et autres Prunières pâlit :

«[...] un disque à mon avis extraordinaire, et dont la perfection rend l'auditeur de plus en plus difficile pour les autres, c'est le Some of These Days, chanté par Sophie Tucker, accompagnée par l'orchestre de Ted Lewis. Je ne connais rien, dans la chanson populaire moderne, qui puisse atteindre à ce cri de désespoir. L'enregistrement de ce disque est admirable, et c'est pour l'avoir entendu que j'écris cet article, en quelque sorte dédié aux chanteuses de couleur dont les belles voix douloureuses, et parfois canailles, rassemblent dans un élan de confession publique tous les Noirs et toutes les "bonnes filles déchues" dont Vaughn de Leath exprime le naïf désespoir. La voix de Sophie Tucker est surprenante. Celui qui écoute le disque et se laisse aller à l'embobinage délicieux du jazz-band sursaute dès le premier appel de cette voix et sent l'émotion l'étreindre à la gorge. Un cri de désespoir monte comme une flamme subitement jaillie des cendres. Cet appel tragique de la race s'échappe, on ne sait comment, des jours les plus anciens de l'esclavage. Ce cri, à lui seul, compose un spectacle plus émouvant que la Revue noire des Champs-Élysées. Retenez le nom de Sophie Tucker, cette belle personne que je ne connais pas et qu'une pointe d'aiguille fait surgir d'un plateau de cire. » (Mac Orlan 1928 : 215-216)

62 La preuve est faite que Roquentin, c'est Mac Orlan. Même attention portée aux accessoires techniques de l'émotion (disques et aiguilles), même jazz-band tout au plus toléré dans l'attente impatiente de la voix, même commotion à l'instant où s'élève cette dernière, même erreur enfin - ici redoublée : Vaughn de Leath est blanche, bien sûr - sur la « race» de la chanteuse... C'est que l'«aiguille» - et c'est son charme - ne «fait surgir » Sophie Tucker qu'incomplètement (ibid. : 216) :

"Quelle est cette chanteuse créatrice d'ondes d'une merveilleuse sélection sentimentale ? Il est difficile pour moi de fixer ses traits. Son aspect varie selon les images qu'elle anime du feu de Prométhée. Car c'est le grand mystère d'un disque de machine parlante que de ne pas nous faire voir le visage de celle ou de celui qui nous émeut dans un secret sentimental plus riche que celui des tombeaux. »

63 Un "grand mystère ", un grand avantage et un joli piège... Point de départ d'un cinéma intérieur éventuellement chargé d'érotisme, le phonographe n'est pas le cinéma. Ce cinéma qui, justement, au moment même où chante Sophie Tucker, devient parlant, avec Al Jolson, autour de cette question du Noir, du Juif, du jazz et de la couleur de la peau...

$\mathrm{Si}$, dans sa rêverie littéraire, Mac Orlan ne commet finalement pas de «fautes » quand Sartre les multiplie, ce n'est donc pas parce qu'il en sait plus long que ce dernier : c'est parce que la structure narrative de son récit - pure fiction assumée et exhibée comme 
telle, portée par un conteur-narrateur omniscient - exclut qu'il fasse des erreurs. En choisissant de donner à La Nausée l'allure d'un journal, Sartre renoue avec cette tradition d'écriture que le critique Jean Rousset a appelée « la fiction du non-fictif » et fait le pari de renouveler le vieux schème du manuscrit trouvé. Mais, en singeant ainsi le document, il s'expose à voir le lecteur - Joë Bousquet, les auteurs des présentes pages... - poser la question de la référence. Dans Sarah au contraire, loin que l'auteur se dissimule derrière la construction d'un artefact, une subjectivité conteuse est au travail qui refuse de se laisser oublier ; en témoigne l'adresse au narrataire : « si vous voulez ».

Ce passage du texte est crucial : s'il est celui où apparaît le titre de la chanson fétiche de Roquentin, c'est aussi le moment où la question de la référence s'avoue comme sans portée. Ted Nicholson ne compose pas Some of These Days, il compose une chanson " chaude et pathétique comme Some of These Days ». Si le song de Shelton Brooks, véritable bande-son de tout un pan de la littérature française des années 1920-1930, confère à l'un et l'autre texte leur atmosphère musicale, Mac Orlan n'en use pourtant que comme un simple comparant. La mention de Some of These Days est certes l'occasion d'en appeler au savoir du lecteur, mais d'un lecteur laissé libre de se figurer pour son propre compte l'air, assurément merveilleux, inventé par Nicholson et chanté par Sarah. Air purement virtuel dont le conte ne citera nulle parole, nulle note. Si le roman est le récit d'une expérience d'écoute, le conte, pour sa part, en appelle à l'expérience d'écoute authentique de son lecteur... Tout en narrant la naissance d'une chanson absolument fictive. Ainsi le réalisme métaphysique de Sartre court-il des risques qui ne menacent pas le réalisme poétique de Mac Orlan.

Sartre n'a probablement lu ni l'article d'Ansermet ni le conte oublié de Mac Orlan, mais il a assurément lu Louis-Ferdinand Céline : rappelons-nous l'épigraphe de La Nausée, extrait de L'Église, publiée en 1933 chez Denoël, et Voyage au bout de la nuit, paru en 1932 chez Gallimard, dont Simone de Beauvoir (1960: 158) dit que "Sartre en prit de la graine » au moment où il recomposa le manuscrit de ce qui allait devenir La Nausée ${ }^{38}$. Dans le Voyage, Céline (1972 : 73), ou plutôt le narrateur Bardamu, note ceci :

« Déjà notre paix hargneuse faisait dans la guerre même ses semences.

On pouvait deviner ce qu'elle serait, cette hystérique rien qu'à la voir s'agiter dans la taverne de l'olympia. En bas dans la longue cave-dancing louchante aux cent glaces, elle trépignait dans la poussière et le grand désespoir en musique négrojudéo-saxonne. Britanniques et Noirs mêlés. Levantins et Russes, on en trouvait partout, fumants, braillants, mélancoliques et militaires, tout du long des sofas cramoisis. "

67 Sans doute est-ce moins l'antisémitisme, le racisme de Céline qui pointe dans cette évocation que - notation ethnomusicologiquement exacte - l'idée du jazz comme musique de mélange, de brassage, de décalage, aux accents à la fois enjoués et désespérés ("mélancoliques et militaires »), due, dans un seul mouvement et tous ensemble, aux Noirs, aux Juifs et aux Anglo-Américains (Séité 1997 : 14-16). Idée que, à peine corrigée, reprendra donc l'auteur de La Nausée. 


\section{BIBLIOGRAPHIE}

ADORNO, Theodor W.

2003 Moments musicaux. Genève, Contrechamps (éd. orig. allemande 1982, trad. Martin Kaltenecker).

Alain

1963 [1926] Système des Beaux-Arts. Paris, Gallimard (« Idées »).

ANSERMET, Ernest

1984 (1919) « Sur un orchestre nègre ", Jazz Magazine $324: 32-33$ et 89.

ARMSTRONG, Louis

2006 [1952] Ma vie à La Nouvelle-Orléans. Paris, Coda (nouvelle trad. franç. par Françoise Thibaut).

AUGÉ, Marc

1992 Non-lieux. Introduction à une anthropologie de la surmodernité. Paris, Seuil.

BADGER, Reid

1995 A Life in Ragtime. A Biography of James Reese Europe. Oxford, Oxford University Press.

BARICCO, Alessandro

1999 Novecento : pianiste. Paris, Les Mille et une nuits (éd. orig. 1994, trad. de l'italien par François Brun).

BAUDOIN, Philippe

1994a « Répertoire », in Philippe Carles, André Clergeat et Jean-Louis Comolli, éds., Dictionnaire du jazz. Paris, Robert Laffont : 994-998.

1994b « Thème », in Philippe Carles, André Clergeat et Jean-Louis Comolli, éds., Dictionnaire du jazz. Paris, Robert Laffont : 1163.

2005 Une chronologie du jazz. Paris, Outre-Mesure.

BEAUVOIR, Simone de

1960 La Force de l'âge. Paris, Gallimard (« Folio »).

BERGEROT, Franck

1996 Miles Davis. Introduction à l'écoute du jazz moderne. Paris, Seuil.

BERRESFORD, Mark

2000 Livret du disque compact Tight Women and Loose Bands. Classic Female Vaudeville Blues Singers with Hot Accompaniments 1921-1931. Wageningen, Timeless Records.

BÉTHUNE, Christian

2003 Adorno et le jazz. Analyse d’un déni. Paris, Klincksieck.

BONNIEUX, Bertrand, CORDEREIX, Pascal, GIULIANI, Élizabeth 
2004 Souvenirs, souvenirs... Cent ans de chanson française. Paris, Gallimard, Bibliothèque nationale de France (« Découvertes »).

BRICKTOP [Ada SMITH DUCONGÉ], HAWKINS, James

1983 Bricktop. New York, Atheneum.

CARLES, Philippe, COMOLLI, Jean-Louis

2000 Free Jazz, Black Power. Paris, Gallimard (« Folio essais »).

CÉLINE, Louis-Ferdinand

1952 [1932] Voyage au bout de la nuit. Paris, Gallimard (« Folio »).

CENDRARS, Blaise

2005 Anthologie nègre, suivi de Petits contes nègres pour les enfants des Blancs, Comment les Blancs sont d'anciens Noirs et de La Création du monde. Paris, Denoël (tome X des CEuvres complètes).

COCTEAU, Jean

2004 [1943] « Danger secret du rythme noir », in Séité $2001:$ 275-280.

Cook, Nicholas

2006 Musique, une très brève introduction. Paris, Allia (éd. orig. anglaise 1998, trad. par Nathalie Gentili).

DESNOS, Robert

1987 Les Voix intérieures. Chansons et textes critiques. Nantes, éd. du Petit Véhicule (textes réunis et préfacés par L. Cantaloube-Ferrieu).

DU BoIs, W.E.B.

1964 [1903] The Souls of Black Folk. Chicago, Fawcett.

ELLISON, Ralph

1964 Shadow and Act. New York, Random House.

FABRI, Franco

2003 « La chanson », in Jean-Jacques Nattiez, éd., Musiques. Une encyclopédie pour le XXe siècle. 1. Musiques du Xx $x^{e}$ siècle. Arles-Paris, Actes Sud, Cité de la musique : 674-702.

FRÉCHET, Philippe

2001 « Trio sartrien : Jean-Paul, Simone et le jazz », Les Cahiers du jazz 1 (nouvelle série, Paris, éd. Alive) : 177-189.

GABBARD, Krim

2002 « The Word Jazz », in Mervyn Cooke et David Horn, éds., The Cambridge Companion of Jazz.

Cambridge, Cambridge University Press, 2002 : 1-6.

GILROY, Paul

2003 L'Atlantique noire. Modernité et double conscience. Paris, Kargo (éd. orig. 1993, traduit de l'anglais [États-Unis] par Jean-Philippe Henquel).

GOFFIN, Robert

1932 Aux frontières du jazz. Paris, éditions du Sagittaire. 
HOLLIER, Denis

1982 Politique de la prose. Jean-Paul Sartre et l'an quarante. Paris, Gallimard.

JACQUES-Charles

1958 La Revue de ma vie. Paris, Librairie Arthème Fayard.

JAMIN, Jean

1996 « Introduction » à Miroir de l’Afrique de Michel Leiris. Paris, Gallimard : 9-59.

1998 « Fausse erreur », L'Homme 146 : 249-263.

2004 « Voix sans issue. À propos de Strange Fruit », L’Homme 170 : 199-230.

2006 « Sonner comme soi-même. Ce que ne nous disent pas les vies de Billie Holiday ", L'Homme $177-178: 179-198$.

JAMIN, Jean, WILLIAMS, Patrick

2001 « Glossaire et index des musiciens de jazz », L’Homme 158-159 : 301-338.

JONES, LeRoi [Amiri Baraka]

1997 Le Peuple du blues. Paris, Gallimard (« Folio essais ») (éd. orig. américaine 1963, trad. par Jacqueline Bernard).

LEIRIS, Michel

1948 Biffures. Paris, Gallimard.

1973 [1939] L’Âge d'homme. Paris, Gallimard (« Folio »).

1988 À cor et à cri. Paris, Gallimard.

1992 Journal 1922-1989. Paris, Gallimard.

LEVET, Jean-Paul

2002 Rire pour ne pas pleurer. Le Noir dans l'Amérique blanche. Marseille, Parenthèses.

2003 Talkin'that talk. Le Langage du blues et du jazz. Paris, Kargo.

LHAMON, William T.

2004 Raising Cain. Représentations du blackface de Jim Crow à Michael Jackson. Paris, Kargo et l'Éclat (éd. orig. américaine 1998, trad. par Sophie Renaut).

LOMAX, Alan

1950 Mister Jelly Roll. The Fortune of Jelly Roll Morton, New Orleans Creole and «Inventor of Jazz ». New York, Duell, Sloan et Pearce (réédition, avec deux nouvelles préfaces de l'auteur [1973 et 1993] et une postface de Lawrence Gushee. Berkeley, University of California Press, 2001).

LORTAT-JACOB, Bernard

2006 « L'image musicale du souvenir. Georgia on My Mind de Ray Charles », L'Homme 177-178 :

$49-72$.

MAC ORLAN, Pierre

1925 « Musiques populaires II », in Aux lumières de Paris. Paris, Georges Crès et Cie : 193-199.

1928 [1927] « Disques I », repris dans « Chronique de la vie sentimentale contemporaine », in Rue Saint-Vincent. Paris, Éditions du Capitole : 209-216. 
1986 [1927] « Sarah », in Manon La Souricière, recueil de contes et nouvelles préfacé par Francis Lacassin. Paris, Gallimard : 51-56.

MARTIN, Denis-Constant, ROUEFF, Olivier

2002 La France du jazz. Musique, modernité et identité dans la première moitié du XXee siècle. Marseille, Parenthèses.

MEZzRow, Milton Mezz, wolfe, Bernard

1966 [1946] La Rage de vivre. Paris, Buchet-Chastel (préface de Henry Miller ; trad. de l'anglais [États-Unis] par Marcel Duhamel et Madeleine Gautier).

MILHAUD, Madeleine et Darius, HOPPENOT, Hélène et Henri

2005 Conversation. Correspondance 1918-1974. Paris, Gallimard.

MOSLEY, Walter

1997 La Musique du diable. Paris, Albin Michel (éd. orig. américaine 1993, trad. par Bernard Cohen). NEVERS, Daniel

1994 « Ragtime », in Philippe Carles, André Clergeat et Jean-Louis Comolli, éds., Dictionnaire du jazz. Paris, Robert Laffont : 973-975.

NIZZA, Angelo

2004 [1934] « Le Voci del jazz. Armstrong in Italia », in Adriano Mazzoletti, éd., Il Jazz in Italia, dalle origini alle grandi orchestre. Turin, EDT : 401-405.

PANASSIÉ, Hugues

1934 Le Jazz hot. Paris, R.-A. Corrêa (présenté par Louis Armstrong, préfacé par Eugène Marsan).

Pénet, Martin, éd.

2001 Mémoire de la chanson. 1200 chansons du Moyen Âge à 1919. Paris, Omnibus.

PERCHERON, Daniel

1994 « Les années Teppaz », Communications 59 : 199-209.

PIERREPONT, Alexandre

2002 Le Champ jazzistique. Marseille, Parenthèses.

POLIAKOV, Léon

1981 Histoire de l'antisémitisme. 2, L'âge de la science. Paris, Seuil (« Points histoire »).

POUCHÈLE, Bernard, LACHAT, Bernard

2006 Le Bruit de fond de l'histoire. Ces chansons qui ont fait la France. Paris, Cheminements.

PRELL, Riv-Ellen

1998 « Appetites : Big Mouth, Big Sex », Lilith Magazine, automne : 136-137.

PRUNIÈRES, Henry

1928 « La musique par disques », La Revue musicale, 9 année, 4 : 85-86.

1929 « La musique par disques », La Revue musicale, 10e année, 3 : 272-275.

ROUEFF, Olivier 
2006 « Politiques d'une "culture nègre". La Revue nègre (1925) comme événement public ", Anthropologie et sociétés (sous presse).

SABATIER, François

2004 La Musique dans la prose française. Des Lumières à Marcel Proust. Paris, Fayard.

SARTRE, Jean-Paul

1938 La Nausée. Paris, Gallimard.

1970 [1947] « Nick's Bar, New York City », in Michel Contat et Michel Rybalka, Les Écrits de Sartre. Paris, Gallimard, 1970 : 680-682.

1979 Le Mur. Paris, Gallimard (« Folio »).

2005 [1946] Réflexions sur la question juive, présenté par Arlette Elkaïm-Sartre. Paris, Gallimard (« Folio essais »).

SCHAEFFER, Jean-Marie

1999 Pourquoi la fiction? Paris, Seuil.

SCHAEFFNER, André

2002 [1927] «Réflexions sur la musique, le jazz », in Martin et Roueff 2002 : 253-256.

SCHAEFFNER, André, CœUROY, André

1988 [1926] Le Jazz. Paris, Jean-Michel Place.

SCHULLER, Gunther

1998 L'Histoire du jazz. Le premier jazz, des origines à 1930. Paris-Marseille, Parenthèses-PUF (éd. orig. américaine 1968, trad. par Danièle Ouzilou).

SEARLE, John

1982 Sens et expression. Paris, Minuit.

SÉITÉ, Yannick

1997 « Ce que le jazz pense de la littérature », Europe 820-821 : 7-22.

1999 « L'Âge du jazz », Gradhiva 25 : 15-44.

2001 «Sur un texte retrouvé de Jean Cocteau », in Mélanges barbares. Hommage à Pierre Michel, Jean-Yves Debreuille et Philippe Régnier, éds. Lyon, PUL : 265-280.

SOUTHERN, Eileen

1976 Histoire de la musique noire américaine. Paris, Buchet-Chastel (éd. orig. américaine 1971, trad. par Claude Yelnick).

sUTTON, Allan

2003 Cakewalks, Rags and Novelties. The International Ragtime Discography (1894-1930). Denver, Mainspring Press.

TOSCHES, Nick

2003 Blackface. Au confluent des voix mortes. Paris, Allia (éd. orig. américaine 2001, trad. par Héloïse Esquié).

TUCKER, Sophie, GILLES, Dorothy 
1945 Some of These Days. The Autobiography of Sophie Tucker. New York, Doubleday.

WATERS, Ethel, SAMUEL, Charles

1952 La Vie en blues. Paris, Robert Laffont (préface de Hugues Panassié, trad. de l'anglais [ÉtatsUnis] par Georges Belmont).

WILLIAMS, Patrick

2005 « Le déni d'Adorno », L’Homme 175-176 : 419-426.

2006 « Standards et standardisation. Sur un aspect du répertoire des musiciens de jazz », L'Homme $177-178: 7-48$

YOUNG-TULIN, Lois

2001 Sophie and Me. Some of These Days. Lincoln (Nebraska), Universe.

\section{ANNEXES}

\section{Chronologie}

1910 : Shelton Brooks compose Some of These Days, paroles et musique. Il présente la chanson à Sophie Tucker qui l'intègre immédiatement à son répertoire.

Le 24 février 1911, Sophie Tucker l'enregistre à New York, avec accompagnement d'orchestre, sur cylindre Edison.

En 1913 Francis Salabert publie la chanson en France avec des paroles françaises de Nilson Fysher et sous le titre Bébé d'amour.

1917 : Arrivée des troupes américaines en France via les ports atlantiques. En novembre, Sartre à La Rochelle.

Début octobre 1918, le 158th U.S. Infantry Band, dirigé par le lieutenant A.R. Etzweiler, enregistre à Paris pour Pathé plusieurs disques à saphir dont The Darktown Strutters'Ball, de Shelton Brooks, et The Story Book Ball, dans lequel apparait le thème principal de Some of These Days.

1921 : Le ténor Robert Jysor enregistre la première interprétation française de la chanson de Shelton Brooks.

Le 23 novembre 1926, Sophie Tucker enregistre à New York une nouvelle version de la chanson avec Ted Lewis and His Orchestra - disque 80 tours Columbia 4269.

1927 : La face remporte en France un immense succès, notamment auprès des « critiques de disques » (Prunières, Desnos, Mac Orlan, Cœuroy, etc.). Le 21 mai, Pierre Mac Orlan publie dans le quotidien Paris Matinal la nouvelle Banana Oil, par la suite rebaptisée Sarah. La firme Pathé commence à enregistrer des 78 tours à aiguille.

À New York, le 10 juillet 1929, accompagnée par Leonard Joy dirigeant un orchestre noir, Sophie Tucker enregistre une nouvelle version de Some of These Days. Ce 78 tours Victor 22049 n'est pas vendu en France.

Février 1931 : Concerts de Sophie Tucker à l'Empire. 
1936 : Sartre entreprend la rédaction de La Nausée.

21 septembre 1937 : Nouvel enregistrement de Some of These Days par Sophie Tucker, avec Harry Sosnik and His Orchestra (78 tours Decca 1472).

1938 : Publication de La Nausée. Lettre de Joë Bousquet signalant à Sartre une ou des « fautes » affectant les pages consacrées à la chanson.

14 octobre 1941 : Aimé Barelli et son orchestre enregistrent Bébé d'amour sur disque Swing.

6 juin 1945 : L'orchestre de Jacques Hélian enregistre Some of This Days (sic) sous le nom de l'accordéoniste Charley Bazin (78 tours Fumière 1034).

30 juin 1947 : Nouvel enregistrement de Some of These Days par Sophie Tucker avec Bob Haggart and His Orchestra (78 tours Decca 24289).

Dans les années 1950, la chanteuse enregistre sur le 45 tours Mercury 5901, avec un orchestre de studio, sa dernière version de Some of These Days.

\section{NOTES}

* Des parties ou versions de cet article ont été présentées, sous forme d'exposés oraux, au colloque "L'Expérience métisse ", dirigé par Serge Gruzinski et organisé par le musée du quai Branly les 2 et 3 avril 2004 à l'auditorium du musée du Louvre, aux séminaires de Daniel Fabre et de Marcello Massenzio au département d'histoire de l'université Tor Vergata de Rome, les 27 et 30 avril 2004, ainsi qu'à la journée d'étude coordonnée par Michel Sandras et Yannick Séité sur le thème " Qu'y a-t-il dans un air ?", qui s'est déroulée dans le cadre du département lettres, art, cinéma à l'université Paris VII le 25 février 2005. Nous remercions les organisateurs et participants à ces manifestations de leurs remarques et suggestions au sujet des thèses qui furent alors développées, et dont nous avons tenu compte pour la rédaction de cet article. Nous remercions également Bernard Lortat-Jacob de ses commentaires et des pistes d'analyse qu'il nous a suggérées, ainsi que Philippe Roussin et Hubert Rolland. Notre gratitude va aussi à Sophie Pécresse pour ses photographies des documents ici insérés, et à Yves Sportis, rédacteur en chef de la revue Jazz Hot, pour son aimable autorisation de reproduire la page de couverture du numéro 227 (janvier 1967) de ladite revue.

1. En jazz, le scat singing désigne une forme de chant se fondant essentiellement sur la vocalisation de syllabes et d'onomatopées qui remplaçent ou prolongent parfois les paroles d'une chanson (Jamin et Williams $2001: 330$ ).

2. L'intégralité de la suite Black, Brown and Beige ne sera enregistrée par l'orchestre de Duke Ellington qu'en février $1958 \mathrm{chez}$ Columbia, avec, pour les parties vocales (IV et VI), la participation de la chanteuse de gospel Mahalia Jackson (version remastérisée et éditée en 1999 sur CD n CK 65566, New York, Sony Music Entertainment Inc.).

3. La première de La Création du monde eut lieu au théâtre des Champs-Élysées le 25 octobre 1923 , exécutée par un ensemble de dix-sept musiciens (Darius Milhaud l'enregistrera plus tard, en 1956, pour la firme d'André Charlin, à la tête d'instrumentistes de l'orchestre du théâtre des Champs-Élysées - version rééditée sur CD en 2005, La Varenne, éditions André-Charlin, ND 217). À noter que la Rhapsody in blue de George Gershwin ou le Concerto en sol de Maurice Ravel, où pointent, comme on sait, les mêmes accents bluesy et jazzy, ne seront composés respectivement qu'en 1924 et 1931, et le Concertino d'Arthur Honegger qu'en 1925. À ce titre, Milhaud fait figure 
de pionnier. Ce qu'a très bien vu André Schaeffner (1988 : 109), même si, probablement égaré par son stravinskysme sourcilleux, il qualifia La Création de Milhaud d'œuvre « de seconde zone ».

4. De son vrai nom Ferdinand Joseph LaMenthe ou LaMothe, devenu Lemott, d'ascendance française, il est issu d'un milieu petit-bourgeois créole de La Nouvelle-Orléans, dont le chef de famille, son beau-père, s'appelait Mouton, d'où probablement l'anglicisme Morton. Voir le récit de vie recueilli par Alan Lomax (1950) à la fin des années 1930, ainsi que le magnifique coffret de 8 CD publié en 2005 par la firme Rounder Records de Washington D.C., comprenant l'intégralité des interviews de Jelly Roll Morton par Alan Lomax à la Library of Congress en 1938, accompagnées de chansons et de pièces jouées en piano solo par le « Doctor Jazz » himself.

5. La bande-son n'est pas sans mièvrerie ni contresens, ainsi en est-il de la scène, pourtant clé, de la joute musicale entre Jelly Roll Morton et Novecento qui caricature le style pianistique stride, lequel, apparu au cours des années 1920 à Harlem, consiste à faire alterner de la main gauche une basse sur les premier et troisième temps de la mesure et un accord dans le registre médium de l'instrument sur les deuxième et quatrième temps, et à jouer de la main droite des tierces, quartes et sixtes qui s'entremêlent dans une improvisation échevelée, le tout sur des tempos généralement très rapides (voirJamin \& Williams $2001: 332$ ).

6. Avec Tim Roth dans le rôle de Novecento, Pruitt Taylor Vince dans celui du narrateur et trompettiste Tim Tooney, et Clarence Williams III (le petit-fils du célèbre pianiste de stride Clarence Williams) dans celui de Jelly Roll Morton.

7. Sur les danses des blackfaces et les spectacles des minstrels qui, malgré l'image insipide qu'en donna Al Jolson en 1927 dans Le Chanteur de jazz (premier film «sonore, parlant et chantant » réalisé par Alan Crosland et produit par la Warner Bros), sont probablement, autant que le blues, le gospel ou le work song, aux origines du jazz, voir les deux études historiques récemment traduites en français : celle de Nick Tosches (2003) et, surtout, celle de William Lhamon (2004). Les minstrels (ou vaudeville) sont une «forme de spectacle apparue [aux États-Unis] vers le milieu du xxe siècle, dans lequel les acteurs blancs s'efforcent d'imiter les représentations données par les esclaves [noirs] pour leurs maîtres [blancs]» (Levet 2003 : 353) et les parodient, le visage grimé de cirage noir ou passé au bouchon brûlé (d'où le nom de blackface).

8. Voir Patrick Williams, "Les paysages du jazz», communication au séminaire de Philippe Descola, « Une anthropologie du paysage est-elle possible? », École des hautes études en sciences sociales, 17 février 2005.

9. Ce qu'à sa manière avait également cru apercevoir Simone de Beauvoir (1960:161): «Comme la plupart des jeunes gens de notre temps, nous étions passionnément émus par les "negro spirituals", par les "chants de travail", par les "blues". Nous aimions pêle-mêle Old Man River, St James Infirmary, Some of These Days, The Man I Love, Miss Hannah, St Louis Blues, Japansy, Blue Sky; la plainte des hommes, leurs joies égarées, les espoirs brisés avaient trouvé pour se dire une voix qui défiait la politesse des arts réguliers, une voix brutalement jaillie du cœur de leur nuit et secouée de révolte ; parce qu'ils étaient nés de vastes émotions collectives [...] - ces chants nous atteignaient chacun en ce point le plus intime de nous-mêmes qui nous est commun à tous; ils nous habitaient, ils nous nourrissaient au même titre que certains mots et certaines cadences de notre propre langue, et par eux l'Amérique existait au-dedans de nous. »

10. Que Some of These Days puisse être par ailleurs considéré comme un standard, cela semble indéniable comme en témoignent les 433 versions du thème répertoriées par Tom Lord dans sa Jazzdiscography. Mais là où standard renvoie à l'appropriation d'un air par les musiciens, aux usages qu'ils en font, tube envisage ce même air sous l'angle de sa réception publique - en l'occurrence triomphale.

11. Mais il importe de souligner que, La Nausée recourant au schème, si sollicité par le xviiie siècle, du manuscrit trouvé, l'avertissement pseudo-éditorial sur lequel s'ouvre le roman date le journal de Roquentin de l'année 1932 (voir Sartre 1938 : 11).

12. Notons les italiques et la graphie du mot, totalement archaïques en 1938. 
13. Datée du 28 mai 1938 et citée par les éditeurs des Euvres romanesques de Jean-Paul Sartre dans la « Bibliothèque de la Pléiade », Michel Contat et Michel Rybalka (Paris, Gallimard, 1981 : 1748). La lettre de Joë Bousquet à Sartre n'a jamais été retrouvée.

14. Jack Hylton (1892-1965), chef d'orchestre et pianiste anglais qui fut un temps considéré comme le Paul Whiteman européen avant de se consacrer exclusivement au théâtre après la Seconde Guerre mondiale. S'il a enregistré Some of These Days en 1933 pour la compagnie Decca, on ne trouve de pot-pourri de ragtimes dans aucun des nombreux medleys - Negro Spiritual Medley, Sousa Marches Medley, etc. - qu'il a laissés au disque.

15. Dans le CD-ROM accompagnant le coffret des enregistrements par Alan Lomax d'entretiens avec Jelly Roll Morton à la Library of Congress en mai 1938, celui-ci précise que (notre traduction) «le ragtime est un genre de musique syncopée, et seuls quelques morceaux pouvaient être joués selon ce principe. Le jazz quant à lui peut s'appliquer à n'importe quel morceau. Mais ce n'est qu'à partir de 1923 que le quatre temps s'est imposé en jazz » (Jelly Roll Morton, The Complete Library of Congress Recordings by Alan Lomax, Washington DC, Rounder Records Corp. 2005 [CD 11661-1892-2], page 214 au format PDF). On peut écouter la fabuleuse démonstration qu'opère Jelly Roll Morton devant Alan Lomax en 1938 (op. cit. 2005, CD n 3, plages 2 et 3) : le pianiste interprète le fameux ragtime de Scott Joplin (1868-1917), Maple Leaf Rag (1899), dans le style Saint Louis d'une part, c'est-à-dire en 2/4, conforme à la partition, et dans le style New Orleans, d'autre part, c'est-à-dire en $4 / 4$, avec réduction de la structure du morceau à son thème principal et accentuation sur les temps faibles de la mesure, ce qui génère du swing et permet l'improvisation, autrement dit, pour reprendre une expression de Jelly Roll Morton, ce qui « donne » le jazz.

16. Ce que suppose également Denis Hollier (1982: 258) lorsqu'il fait allusion au déménagement des parents de Sartre à La Rochelle en 1917. C'est après ce déménagement que le jeune Sartre se serait remis à jouer du piano et il est possible de l'imaginer, comme le fait Hollier, jouant à quatre mains avec sa mère la partition de Some of These Days, que les éditions Salabert avaient du reste publiée en France en 1913.

17. On notera qu'il juxtapose, dans un ordre discursif non chronologique, quatre voire cinq («à saphir » vaut mention temporelle) temporalités en deux lignes : "Je l'ai entendu siffler en 1917 [...]. Il doit dater d'avant-guerre. Mais l'enregistrement est beaucoup plus récent. Tout de même, c'est le plus vieux disque de la collection, un disque Pathé pour aiguille à saphir» (souligné par nous).

18. Il y remporta un premier prix pour son interprétation de My Ragtime Baby, composition écrite en 1898 par le pianiste noir Fred Stone. Ce furent ces concerts qui donnèrent à la France un premier aperçu de la musique de ragtime (Southern 1976 : 245 ; Baudouin 2005 : 31). Rappelons que Claude Debussy, qui fut un des visiteurs et des auditeurs assidus de l'Exposition universelle de 1900, compte parmi les premiers compositeurs européens de musique savante (avant le célèbre Rag-time pour onze instruments d'Igor Stravinsky composé en 1918) à s'être inspiré du ragtime, que ce soit dans les toutes premières mesures de Mouvement du livre I des Images (1905), dans Le Petit Nègre (1909) ou dans les six pièces pour piano baptisées Children's Corner (1906-1908), notamment dans la sixième d'entre elles, évoquant la danse d'un polichinelle: Golliwogg's Cake Walk. Le cake walk (litt. danse du gâteau) désignait un pas de danse syncopé inventé par les esclaves noirs des plantations du Sud, peut-être inspiré des danses de guerre des Indiens Seminole, puis repris par les artistes blancs des minstrels dans leurs spectacles.

19. Voir Milton Mezz Mezzrow et Bernard Wolfe (1966: 80-81) : «La plupart des artistes en renom de cette époque [années 1920]: Ted Lewis, Sophie Tucker, Benny Davis, Eddie Cantor, Dolly Kaye, Al Jolson (qu'on avait même glorifié du nom de "Chanteur de jazz") étaient tous des youds et les copains étaient d'avis qu'il fallait se tenir les coudes et ne pas débiner les célébrités de "notre" race. " 
20. C'est-à-dire Absent WithOut Leave, abréviation usitée dans le jargon militaire anglo-saxon et qui peut se traduire par : " porté manquant à l'appel ».

21. Coon-shouter: de coon (litt. raton-laveur), terme péjoratif désignant les Noirs dans le Sud, et de shouter, chanteur au style expressif et puissant, dont l'équivalent en français serait peut-être mieux rendu par l'expression "chanter à tue-tête ». Les coon-shouters désignent surtout des interprètes blancs qui caricaturent le parler, les intonations et les attitudes des Noirs. Des troupes de minstrels vinrent très tôt en Europe et, notamment, en France, dès les années 1880 et remportèrent un vif succès, se produisant surtout aux Folies-Bergère, comme les Bellonini Brothers à partir de 1885, rebaptisés pour le public français « Les Hottentots à l'œil blanc »!

22. Voir sur cette question le remarquable roman de Richard Powers, récemment traduit en français : Le temps où nous chantions (Paris, Le Cherche-Midi, 2006).

23. Et prend parfois l'allure d'un pur et simple révisionnisme; voir le livre de Richard M. Sudhalter, Lost Chords: White Musicians and Their Contribution to Jazz, 1915-1945. New York, Oxford University Press, 1999.

24. Tight Women and Loose Bands. Classic Female Vaudeville Blues Singers with Hot Accompaniments 1921-1931, Timeless Records, 2000.

25. Qui dans son premier livre Le Jazz hot note, au terme de son paragraphe sur Bessie Smith : «C'est d'elle que s'est inspirée la fameuse chanteuse blanche, Sophie Tucker.» (Panassié 1934 : 175)

26. C'est le moment de rappeler que, dès 1913, l'éditeur de musique Francis Salabert avait acheté les droits de Some of These Days et avait commandé à Alfred Nilson-Fysher - très intéressante figure cosmopolite de cabaretier - des paroles françaises. C'est ainsi que Some of These Days devint Bébé d'amour, romance. L'historien de la chanson Martin Pénet (2001) recense deux interprétations sur disque de Bébé d'amour: celle du ténor Robert Jysor en 1921 et celle, plus tardive, de Fred Gouin en 1933. Bien qu'elle soit seulement instrumentale, la plus intéressante musicalement et historiquement est due au trompettiste Aimé Barelli. On sait que les jazzmen parisiens crurent prudent de travestir de titres français de fantaisie les chansons américaines qu'ils enregistrèrent durant l'Occupation. Or, s'agissant de Some of These Days, nul besoin d'en user comme avec Honeysuckle Rose, devenu Chèvrefeuille: le thème existait déjà sous un titre français et c'est un bien innocent mais très swinguant Bébé d'amour que Barelli enregistre pour la firme Swing en octobre 1941. On peut voir dans le fait que l'accordéoniste Charley Bazin ait restitué au thème son titre originel, dans son enregistrement pour Fumières en juin 1945, un indice de la liberté retrouvée.

27. Chanson composée en 1929 par Jack Yellen et Milton Alger. Elle servit d'hymne à la convention démocrate de 1932 et scanda la campagne de Franklin D. Roosevelt pour l'élection présidentielle.

28. Dans son livre de souvenirs sur Sophie Tucker, Lois Young-Tulin (2001: 108-109) signale qu'en 1933, dès son avènesment au pouvoir, Hitler avait donné l'ordre de détruire et d'interdire de vente sur tout le territoire allemand les enregistrements de My Yiddishe Momme. Indignée, Sophie Tucker lui écrivit "pour lui dire son fait », mais, ajoute-t-elle, sa lettre demeura sans réponse...

29. Rythme à quatre temps avec accentuation des temps faibles $\left(2^{\mathrm{e}}\right.$ et $\left.4^{\mathrm{e}}\right)$, mélodie tendue, tierce et septième mineures, quinte augmentée, voix projetée au vibrato appuyé, et légèrement décalée par rapport au tempo, attaques sur le contretemps, etc. - traits musicaux auxquels le public français était devenu sensible et auxquels il s'était familiarisé depuis, surtout, la représentation de la Revue nègre en 1925 au théâtre des Champs-Élysées, laquelle avait non seulement fait les choux gras des magazines de critique musicale et culturelle de l'époque (Martin et Roueff 2002 ; Roueff 2006) mais séduit des écrivains, artistes et intellectuels (Jamin 1996 ; Séité 1999).

30. Cette version de Some of These Days a été reprise sur le double CD Louis Armstrong, The Quintessence. New York-Chicago, 1923-1946 (Paris, Frémeaux et Associés, 1997, nº FA 221). Hugues 
Panassié (in Martin et Roueff 2002 : 288) la considérera comme une des plus grandes réussites du musicien. Sophie Tucker, quant à elle, enregistra six versions de Some of These Days. Elle la chanta, en particulier, dans deux films : Broadway Melody of 1938, réalisé en 1937 par Roy Del Ruth pour les studios MGM ; Follow the Boys (sorti en France sous le titre Hollywood Parade), réalisé en 1944 par Eddie Sutherland pour Universal Pictures.

31. À noter que c'est avec ce passage de l'autobiographie de Sophie Tucker - passage alors cité en entier - que Denis Hollier (1982 : 11-12) ouvre son bel essai sur Sartre.

32. Compositeur et parolier, Stephen Collins Foster (1826-1864), natif de Pittsburgh, à qui Walt Whitman et Abraham Lincoln, entre autres, vouèrent une véritable admiration, fut le premier Américain à composer pour des troupes de blackfaces et à gagner sa vie en écrivant des chansons qui comptent parmi les plus populaires et les plus éternelles de la culture américaine, telles que Old Black Joe, Oh! Susannah, My Old Kentucky Home, The Old Folk at Home, etc. (voir Tosches 2003 : 21-23).

33. André Francis et Jean Schwarz le reproduisent dans leur superbe anthologie du jazz vocal (plage 1 du CD 1, Histoire du jazz vocal, vol. I, 1911-1940, Le Chant du monde, Harmonia mundi, n - 574 1301.10), sans indication du nom de l'orchestre. Dans cette interprétation, on peut déceler encore l'influence du minstrel show sur le style vocal et le tempo utilisés par la chanteuse (couplets déclamés en parlando, interpellations, entrée du refrain sur un trois temps alors que la rythmique de l'orchestre joue en deux temps, voix au vibrato parfois appuyé, suspension du rythme) qui tranchent avec la version enregistrée quinze ans plus tard, nettement plus coulée, rapide et syncopée, plus projetée et vocalisée, et où l'attaque se fait sur le contretemps. Sur la composition de l'orchestre aussi (incessantes ponctuations de piccolos; pas de batterie mais des tambours...). Pour sa part, David Wondrich, l'auteur de Stomp and Swerve : American Music Gets Hot 1843-1924, qui a également réédité le cylindre dans le disque éponyme qui accompagne son livre (Archeophone 1003), le commente en ces termes (p. 20 du livret d'accompagnement, notre traduction) : « C'est une plainte inoubliable, low-down, bouleversante, qui entretisse le sens de l' écart africain et la mélancolie ashkénaze. "

34. Dans le texte de pochette du disque de George McClennon : Harlem Trio, George McClennon Jazz Devils + Wilton Crawley (remaining titles), recorded in New York, 1923-1930, Jazz Oracle BDW 8022, Toronto et Burnham, 2001.

35. Dont on signalera la cruelle plaisanterie: "Ted Lewis could make the clarinet talk. What it said was "put me back in the case!" (" Ted Lewis savait faire parler la clarinette. Elle disait: "Remetsmoi dans mon étui !" ")

36. Sur "Dinah, fille de couleur déracinée ", voir aussi Mac Orlan 1928: 212-213. C'est à l'interprétation de Dinah par le groupe vocal des Revellers, virtuoses du chant harmonisé, que Mac Orlan se réfère dans ce texte.

37. Le parallélisme des destins des peuples noir et juif explique sans doute l'absence, dans la littérature française des années 1920-1930 et au-delà, de toute mention de la communauté italienne comme ayant joué un rôle essentiel dans le développement de la musique populaire américaine. C'est que l'émigration italienne est une émigration économique, dont les causes sont moins dramatiques que l'esclavage ou les pogroms. Les critiques et écrivains du début du xxe siècle, qui savent que "les plus désespérés sont les chants les plus beaux », sont si bien informés de la présence des Juifs dans la musique américaine qu'ils font de cette présence un objet de littérature. Personne en revanche pour signaler la dette immense contractée par le jazz envers les Italo-Américains; personne pour faire de Nick La Rocca, Frank Guarente, Leon Ròppolo (dit Rappolo), George Vitale (dit «Papa » Jack Laine) ou Wingy Manone des personnages littéraires. D'où l'intérêt aujourd'hui d'un livre comme celui de Ronald L. Morris, Le Jazz et les gangsters 1880-1940, trad. franç. par Jacques B. Hess. Paris, éditions Abbeville, 1997 (voir également Jamin 1998). 
38. Dans le passage de La Nausée sur le «Juif aux sourcils de charbon », un tour comme "Ça s'est passé comme ça » renvoie explicitement, sur un plan stylistique, au négligé savant de la phrase liminaire du Voyage : "Ça a débuté comme ça. »

\section{RÉSUMÉS}

À partir de la musique de jazz et de l'un de ses plus célèbres thèmes, cet article se propose d'explorer cette sorte d'«épopée de la chose " que sont, selon la belle formule du philosophe Alain, musique et chant, et où ce qui s'exprime dans leur apparente contingence est non seulement une modulation mais un modelage du rapport de soi à soi, et de soi à l'autre. Sujet bien évidemment anthropologique qui sera abordé à travers l'étude de trois œuvres d'écrivains du xx siècle (Pierre Mac Orlan, Jean-Paul Sartre, Alessandro Baricco). Le "chant du jazz », que toutes trois placent au premier plan, traduit une dialectique de l'identité et de l'altérité en jouant sur des associations et des contrastes de couleurs tout autant musicales que charnelles. À l'image de la célèbre suite Black, Brown and Beige que Duke Ellington créa en 1943, vingt ans plus tôt Darius Milhaud avait voulu les restituer dans la musique de ballet La Création du monde en combinant jazz, blues et traditions musicales européennes savantes, le tout joué et dansé dans un décor conçu par Fernand Léger, aux longs aplats de couleur, en noir, blanc et ocre. Nous sommes ici, avec ces trois œuvres, en noir, blanc et blond.

Starting with jazz and one of its best known themes, the article sets out to explore that kind of " epic of the thing " that, as the philosopher Alain so aptly phrases it, music and song constitute, and where what is expressed in their apparent contingence is not only a modulation but also a modelling of the relationship between self and self and between self and the other. This is clearly an anthropological subject, which is approached through a study of three works by 20th century writers (Pierre Mac Orlan, Jean-Paul Sartre and Alessandro Baricco). The "jazz singing », which all three focus upon, expresses a dialectic of identity and otherness, playing on associations and contrasts of colours, both musical and fleshly. As the famous Black, Brown and Beige suite created by Duke Ellington in 1943, Darius Milhaud sought to recreate them twenty years sooner in his music for the ballet The Creation of the World, combining jazz, blues and scholarly European musical traditions, all played and danced in a set designed by Fernand Léger, composed of long black, white and ochre flats. Here, with these three works, our colours are black, white and blond.

\section{INDEX}

Mots-clés : chansons, ethnomusicologie, jazz, musique et littérature, vaudeville

Keywords : ethnomusicology, jazz, minstrel show, music and literature, songs

\section{AUTEURS}

JEAN JAMIN

École des hautes études en sciences sociales, LAHIC, jamin@ehess.fr 


\section{YANNICK SÉITÉ}

Université Paris VII, UFR Lettres, Arts, Cinéma, équipe Théorie de la littérature et sciences

humaines, seite@ccr.jussieu.fr 